\title{
Multiscale semi-Lagrangian lattice Boltzmann method
}

\author{
N. G. Kallikounis, B. Dorschner ๑, and I. V. Karlin $\odot^{*}$ \\ Department of Mechanical and Process Engineering, ETH Zurich, 8092 Zurich, Switzerland
}

(Received 24 February 2021; revised 14 May 2021; accepted 20 May 2021; published 7 June 2021)

\begin{abstract}
We present a multi-scale lattice Boltzmann scheme, which adaptively refines particles' velocity space. Different velocity sets of lower and higher order are consistently and efficiently coupled, allowing us to use the higher-order model only when and where needed. This includes regions of high Mach or high Knudsen numbers. The coupling procedure of discrete velocity sets consists of either a projection of the higher-order populations onto the lower-order lattice or lifting of the lower-order populations to the higher-order velocity space. Both lifting and projection are local operations, which enable a flexible adaptive velocity set. The proposed scheme is formulated for both a static and an optimal, co-moving reference frame, in the spirit of the recently introduced Particles on Demand method. The multi-scale scheme is validated with an advection of an athermal vortex and in a jet flow setup. The performance of the proposed scheme is further investigated in the shock structure problem and a high-Knudsen-number Couette flow, typical examples of highly non-equilibrium flows in which the order of the velocity set plays a decisive role. The results demonstrate that the proposed multi-scale scheme can operate accurately, with flexibility in terms of the underlying models and with reduced computational requirements.
\end{abstract}

DOI: 10.1103/PhysRevE.103.063305

\section{INTRODUCTION}

With its roots in kinetic theory, the lattice Boltzmann Method (LBM) describes fluid flow via the propagation and collision of discretized particle distribution functions (populations), which are associated with a set of discrete velocities and constructed to recover the macroscopic Navier-Stokes equations (NSE) in the hydrodynamic limit. LBM has matured to a competitive alternative to conventional numerical solvers, with a vast range of applications including compressible flows [1], complex moving geometries [2], multiphase flows $[3,4]$ and rarefied gas dynamics [5], to mention a few.

The most common LBM models use so-called standard lattices such as the $D 2 Q 9$ or the $D 3 Q 27$ in two or three dimensions $(D=2,3)$ with $Q=9$ and $Q=27$ discrete velocities, respectively. While this is mainly due to their simplicity and efficiency, the limited number of speeds puts severe restrictions on their range of validity. On the other hand, a systematic increase of the number of velocities to so-called high-order or multispeed lattices has been shown to extend the range of validity significantly. High-order lattices can be constructed systematically either by discretizing the Boltzmann equation on the roots of the Hermite polynomials $[5,6]$ or by entropy considerations, yielding a set of so-called admissible lattices $[7,8]$. Note that the roots of the Hermite polynomials are irrational numbers and thus require off-lattice propagation

\footnotetext{
*Corresponding author: ikarlin@ethz.ch
}

Published by the American Physical Society under the terms of the Creative Commons Attribution 4.0 International license. Further distribution of this work must maintain attribution to the author(s) and the published article's title, journal citation, and DOI. schemes such as the semi-Lagrangian LBM $[9,10]$, whereas admissible lattices as in $[7,8]$ remain on-lattice with integervalued velocities. The increase of accuracy of such lattices can be exploited in many applications such high-speed flows, non-equilibrium gas flows or relativistic fluids $[1,11-13]$ to name a few.

In this paper, we will restrict our attention to compressible as well as non-equilibrium flows but the proposed concepts are generic and can be used for all multi-scale applications using high-order lattices. In particular, it is well known that the lack of Galilean invariance and insufficient isotropy of standard lattices, limits classical LB models to isothermal, low-Mach number flows $[14,15]$ and the extension of LBM to high-speed compressible flows is still an active area of investigation. For instance, so-called augmented and extended LB models have been developed in [16-19] to mitigate these shortcomings by introducing non-local corrections into the kinetic equations to eliminate the error terms in the momentum and energy equations, which arise due to the constraints of the standard lattices. Promising results have been shown in recent contributions, featuring both variable Prandtl number and adiabatic exponent [17]. Moreover, the recently proposed Particles on Demand (PonD) method [20,21] eliminates the Galilean invariance errors of the standard lattices from the outset by representing the populations in a co-moving reference frame. Note that while both of these approaches get by with minimal velocity sets, another alternative to lift the aforementioned constraints is the use of multispeed lattices. There, the increase of the number of speeds moderates the lattice constraints and pertinent moments to recover the full Navier-Stokes-Fourier (NSF) equations in the hydrodynamic limit can be represented by the lattice [22-25]. It must be noted however that while multispeed lattices can extend the range of velocity significantly, the associated temperature 
range typically decreases with an increase of the number of particle's velocities. Recently, an entropic LBM realization of multispeed lattices has demonstrated promising results for both trans- and supersonic flows [1].

High-order lattices can also be used to increase accuracy in non-equilibrium flows. The degree of non-equilibrium or rarefaction is usually quantified by the Knudsen number, which is defined as the ratio of the molecular mean free path and a characteristic length. It has been shown both analytically and numerically $[26,27]$ that by increasing the number of discrete velocities (order of Gauss-Hermite quadrature), LB models can capture non-equilibrium effects of wall-bounded flows beyond the NSF level.

With the examples from above in mind, it needs to be mentioned that while high-order lattices can provide a more accurate description of the flow, they come at high computational costs, which can make these models prohibitive for flows with realistic complexity in three dimensions. Fortunately, for most practical applications, the regions requiring high-order velocity sets are typically confined to a small subregion of the entire computational domain. Hence, significant computational resources can be saved by using a multi-scale description, which uses higher-order lattices only when and where needed. In that spirit, a variety of different multi-scale frameworks, coupling different methods have been proposed in the literature [28]. For example, a multi-scale model, coupling LBM for continuum regions to direct simulation Monte Carlo (DSMC) for the high Knudsen number regions, was proposed in $[29,30]$ for steady-state simulations. Furthermore, so-called discrete velocity models (DVM) have been shown to be successful in simulating rarefied gases [31-33] and multi-scale schemes with adaptively refined phase space meshes have been proposed to reduce both computational time and memory [34,35]. DVM have also been coupled with the more efficient LBM, which was used in continuum flow zones whereas the DVM was restricted to the rarefied regions only $[36,37]$. Finally, in the realm of LBM, a finite difference LB scheme for rarefied gas dynamics was proposed in [38], where different lattices are coupled in a static manner by a non-local extrapolation procedure. While interesting, this approach is limited to static phase space refinement and suffers from severe stability issues due to a non-local ad hoc coupling procedure of different lattices based on extrapolation procedures.

In this work, we propose a multi-scale LBM scheme, which alleviates these issues and allows for adaptive velocity space refinement using a consistent and local coupling procedure. For maximum lattice flexibility, we use a semi-Lagrangian advection procedure to naturally decouple the velocity space from the physical space [9,20,39-42]. Higher-order lattices are thus only used when and where necessary, while preserving second-order accuracy. We further shed light on the nature of multi-scale problems and the range of validity of coupling procedures. The proposed scheme is then validated on examples in both high-Mach and high-Knudsen number flows but the scheme can be beneficial whenever large lattices are needed in a confined region. For illustration of the coupling procedure, we use a dual population, multispeed LBM model with variable Prandtl number and adiabatic exponent as proposed in [1] for high-Mach regions. Further, while high-Mach
TABLE I. Lattice temperature $T_{L}$, roots of Hermite polynomials $c_{i \alpha}$ and weights $w_{i \alpha}$ of the $D=1$ Gauss-Hermite quadrature, and nomenclature.

\begin{tabular}{lcccc}
\hline \hline Model & $T_{L}$ & $c_{i \alpha}$ & $w_{i \alpha}$ & $D=2$ \\
\hline$D 1 Q 3$ & 1 & 0 & $2 / 3$ & $D 2 Q 9$ \\
& & $\pm \sqrt{3}$ & $1 / 6$ & \\
$D 1 Q 4$ & 1 & $\pm \sqrt{3-\sqrt{6}}$ & $(3+\sqrt{6}) / 12$ & $D 2 Q 16$ \\
& & $\pm \sqrt{3+\sqrt{6}}$ & $(3-\sqrt{6}) / 12$ & \\
$D 1 Q 5$ & 1 & 0 & $8 / 15$ & $D 2 Q 25$ \\
& & $\pm \sqrt{5-\sqrt{10}}$ & $(7+2 \sqrt{10}) / 60$ & \\
& & $\pm \sqrt{5+\sqrt{10}}$ & $(7-2 \sqrt{10}) / 60$ & \\
\hline \hline
\end{tabular}

number flows are intrinsically captured in PonD through an adaptive reference frame, we extend PonD's range of validity to non-equilibrium flows using multispeed lattices.

The paper is organized as follows. In Sec. II the twopopulation model is presented and its semi-Lagrangian realization is explained. Subsequently, in Sec. III the multi-scale coupling scheme is introduced. In Sec. IV, a Taylor-Green vortex setup is simulated for validation and convergence order study. Further numerical results include simulations of an athermal advected vortex, a jet flow, the shock structure problem and a high Knudsen Couette flow. The computational efficiency of the coupling scheme is additionally analysed. Finally, concluding remarks are given in Sec. V.

\section{MODEL DESCRIPTION}

\section{A. Discrete velocities}

Without a loss of generality, we consider discrete speeds in two dimensions, $D=2$, formed by tensor products of roots of Hermite polynomials $c_{i \alpha}$,

$$
\boldsymbol{c}_{i}=\left(c_{i x}, c_{i y}\right) .
$$

The roots of the lowest three Hermite polynomials are collected in Table I for the sake of completeness. Following the standard nomenclature, we refer to the corresponding discrete speeds (1) as $D 2 Q 9, D 2 Q 16$ and $D 2 Q 25$ models. Each model is characterized by the lattice temperature $T_{L}$ and the weights $W_{i}$ associated with the vectors (1),

$$
W_{i}=w_{i x} w_{i y},
$$

where $w_{i \alpha}$ are weights of the Gauss-Hermite quadrature (see Table I). Note that the lattice temperature is set to $T_{L}=1$, for all the models under consideration.

With the discrete speeds (1), the particles' velocities $\boldsymbol{v}_{i}$ are defined relative to a reference frame, specified by the frame velocity $\boldsymbol{u}_{\text {ref }}$ and the reference temperature $T_{\text {ref }}$,

$$
\boldsymbol{v}_{i}=\sqrt{\frac{R T_{\mathrm{ref}}}{T_{L}}} \boldsymbol{c}_{i}+\boldsymbol{u}_{\mathrm{ref}},
$$

where $R$ is the gas constant. Two reference frames of interest shall be considered below. The local co-moving reference frame is specified by the local temperature $T=T(\boldsymbol{x}, t)$ and 
the local flow velocity $\boldsymbol{u}=\boldsymbol{u}(\boldsymbol{x}, t)$. The lattice reference frame is specified by $\boldsymbol{u}_{\text {ref }}=\mathbf{0}$ and $T_{\text {ref }}=T_{L} / R$.

\section{B. Kinetic equations}

For the sake of presentation, we consider a two-population kinetic model for ideal gas with a variable adiabatic exponent and Prandtl number [1],

$$
\begin{aligned}
& f_{i}(\boldsymbol{x}, t)-f_{i}\left(\boldsymbol{x}-\boldsymbol{v}_{i} \delta t, t-\delta t\right) \\
& \quad=\omega_{1}\left(f_{i}^{\mathrm{eq}}-f_{i}\right)+\left(\omega_{1}-\omega_{2}\right)\left(f_{i}^{*}-f_{i}^{\mathrm{eq}}\right), \\
& g_{i}(\boldsymbol{x}, t)-g_{i}\left(\boldsymbol{x}-\boldsymbol{v}_{i} \delta t, t-\delta t\right) \\
& \quad=\omega_{1}\left(g_{i}^{\mathrm{eq}}-g_{i}\right)+\left(\omega_{1}-\omega_{2}\right)\left(g_{i}^{*}-g_{i}^{\mathrm{eq}}\right),
\end{aligned}
$$

where $f_{i}^{\mathrm{eq}}$ and $g_{i}^{\mathrm{eq}}$ are local equilibria while $f_{i}^{*}$ and $g_{i}^{*}$ are quasi-equilibrium populations. Moreover, $\delta t$ is the time step and $\omega_{1}$ and $\omega_{2}$ are relaxation parameters related to the dynamic viscosity and thermal conductivity [1], respectively. The local conservation laws for the density $\rho$, momentum $\rho \boldsymbol{u}$ and the total energy $\rho E$ are,

$$
\begin{gathered}
\rho=\sum_{i=0}^{Q-1} f_{i}=\sum_{i=0}^{Q-1} f_{i}^{\mathrm{eq}}, \\
\rho \boldsymbol{u}=\sum_{i=0}^{Q-1} \boldsymbol{v}_{i} f_{i}=\sum_{i=0}^{Q-1} \boldsymbol{v}_{i} f_{i}^{\mathrm{eq}}, \\
\rho E=\sum_{i=0}^{Q-1} \frac{v_{i}^{2}}{2} f_{i}+\sum_{i=0}^{Q-1} g_{i}=\sum_{i=0}^{Q-1} \frac{v_{i}^{2}}{2} f_{i}^{\mathrm{eq}}+\sum_{i=0}^{Q-1} g_{i}^{\mathrm{eq}} .
\end{gathered}
$$

We consider ideal gas with the internal energy $U=C_{v} T$, where $C_{v}$ is the specific heat at constant volume. The total energy is,

$$
\rho E=C_{v} \rho T+\frac{\rho u^{2}}{2} .
$$

The quasi-equilibrium populations are,

$$
\begin{gathered}
f_{i}^{*}=f_{i}^{\mathrm{eq}}+W_{i} \frac{\overline{\boldsymbol{Q}}:\left(\theta^{3 / 2} \boldsymbol{c}_{i} \otimes \boldsymbol{c}_{i} \otimes \boldsymbol{c}_{i}-R T \theta^{1 / 2} \operatorname{sym}\left(\boldsymbol{c}_{i} \otimes \mathbf{1}\right)\right)}{6(R T)^{3}}, \\
g_{i}^{*}=g_{i}^{\mathrm{eq}}+W_{i} \frac{\theta^{1 / 2} \overline{\boldsymbol{q}} \cdot \boldsymbol{c}_{i}}{R T},
\end{gathered}
$$

where $\operatorname{sym}(\ldots)$ denotes symmetrization, ":" indicates full contraction and $\theta$ is the reduced temperature,

$$
\theta=\frac{R T}{T_{L}},
$$

while the non-equilibrium third-order tensor $\bar{Q}$ and the heat flux vector $\overline{\boldsymbol{q}}$ are,

$$
\begin{gathered}
\overline{\boldsymbol{Q}}=\sum_{i=0}^{Q-1}\left(\boldsymbol{v}_{i}-\boldsymbol{u}\right) \otimes\left(\boldsymbol{v}_{i}-\boldsymbol{u}\right) \otimes\left(\boldsymbol{v}_{i}-\boldsymbol{u}\right)\left(f_{i}-f_{i}^{\mathrm{eq}}\right), \\
\overline{\boldsymbol{q}}=\sum_{i=0}^{Q-1}\left(\boldsymbol{v}_{i}-\boldsymbol{u}\right)\left(g_{i}-g_{i}^{\mathrm{eq}}\right) .
\end{gathered}
$$

When the local flow velocity $\boldsymbol{u}(\boldsymbol{x}, t)$ and the local temperature $T(\boldsymbol{x}, t)$ are used to gauge the particles' velocities (3),

$$
\boldsymbol{u}_{\mathrm{ref}}=\boldsymbol{u}(\boldsymbol{x}, t), \quad T_{\mathrm{ref}}=T(\boldsymbol{x}, t),
$$

we say that kinetic equations (4) and (5) are formulated in the co-moving reference frame, where the equilibrium populations depend only on the density and the temperature,

$$
\begin{gathered}
f_{i}^{\mathrm{eq}}=\rho W_{i}, \\
g_{i}^{\mathrm{eq}}=\left(C_{v}-\frac{D}{2} R\right) T \rho W_{i} .
\end{gathered}
$$

With the co-moving reference frame and for any of the models of Sec. II A, the hydrodynamic limit of the kinetic equations (4) and (5) are the standard equations of compressible gas dynamics, with the dynamic viscosity $\mu$, thermal conductivity $\kappa$ and the bulk viscosity $\xi$ related to the relaxation parameters $\omega_{1}$ and $\omega_{2}$ as follows,

$$
\begin{gathered}
\mu=\left(\frac{1}{\omega_{1}}-\frac{1}{2}\right) \rho R T \delta t, \\
\kappa=C_{p}\left(\frac{1}{\omega_{2}}-\frac{1}{2}\right) \rho R T \delta t, \\
\xi=\left(\frac{1}{C_{v}}-\frac{2}{D R}\right) \mu .
\end{gathered}
$$

Here $C_{p}$ is the specific heat of ideal gas at constant pressure, $C_{p}=C_{v}+R$. The Prandtl number is defined as $\operatorname{Pr}=C_{p} \mu / \kappa$, and the adiabatic exponent is $\gamma=C_{p} / C_{v}$. In the following, we set $R=1$, without loss of generality.

\section{Semi-Lagrangian realization}

\section{Co-moving reference frame}

The implementation of the propagation using the comoving reference frame requires a transformation between non-equal reference frames which we remind for the sake of completeness [20]. A reference frame $\lambda$ is defined by

$$
\lambda=\{\boldsymbol{u}, T\},
$$

with the particles velocities

$$
\boldsymbol{v}_{i}^{\lambda}=\sqrt{\theta} \boldsymbol{c}_{i}+\boldsymbol{u}
$$

In a given reference frame $\lambda$, the $Q$ linearly independent moments of the $f$-and of the $g$-populations are defined as,

$$
\begin{aligned}
M_{m n}^{\lambda} & =\sum_{i=0}^{Q-1} f_{i}^{\lambda}\left(\sqrt{\theta} c_{i x}+u_{x}\right)^{m}\left(\sqrt{\theta} c_{i y}+u_{y}\right)^{n}, \\
N_{m n}^{\lambda} & =\sum_{i=0}^{Q-1} g_{i}^{\lambda}\left(\sqrt{\theta} c_{i x}+u_{x}\right)^{m}\left(\sqrt{\theta} c_{i y}+u_{y}\right)^{n},
\end{aligned}
$$

where $m, n \in\{0, \ldots, \sqrt{Q}-1\}$. Equations (23) and (24) establish a linear map of the $Q$-dimensional population vectors $f^{\lambda}$ and $g^{\lambda}$ into the moment vectors $M^{\lambda}$ and $N^{\lambda}$. Denoting $\mathcal{M}_{\lambda}$ as the $Q \times Q$ the matrix of this map, we write Eqs. (23) and (24) as,

$$
\begin{aligned}
& M^{\lambda}=\mathcal{M}_{\lambda} f^{\lambda}, \\
& N^{\lambda}=\mathcal{M}_{\lambda} g^{\lambda} .
\end{aligned}
$$


Consider two reference frames, $\lambda$ and $\lambda^{\prime}$. Following [20], populations are transformed based on the principle of independence of the moments on the reference frame,

$$
\begin{aligned}
M^{\lambda} & =M^{\lambda^{\prime}}, \\
N^{\lambda} & =N^{\lambda^{\prime}} .
\end{aligned}
$$

With Eqs. (27) and (28), the populations are transformed from the reference frame $\lambda$ to the reference frame $\lambda^{\prime}$,

$$
\begin{aligned}
& f^{\lambda^{\prime}}=\mathcal{G}_{\lambda}^{\lambda^{\prime}} f^{\lambda}, \\
& g^{\lambda^{\prime}}=\mathcal{G}_{\lambda}^{\lambda^{\prime}} g^{\lambda},
\end{aligned}
$$

where the transfer matrix $\mathcal{G}_{\lambda}^{\lambda^{\prime}}$ reads,

$$
\mathcal{G}_{\lambda}^{\lambda^{\prime}}=\mathcal{M}_{\lambda^{\prime}}^{-1} \mathcal{M}_{\lambda}
$$

Finally, populations are reconstructed at a point $\boldsymbol{x}$ and time $t$ using Lagrange interpolation,

$$
\begin{aligned}
& \bar{f}_{i}^{\lambda}(\boldsymbol{x}, t)=\sum_{s=1}^{p} a_{s}\left(\boldsymbol{x}-\boldsymbol{x}_{s}\right) \mathcal{G}_{\lambda_{s}}^{\lambda} f^{\lambda_{s}}\left(\boldsymbol{x}_{s}, t\right), \\
& \bar{g}_{i}^{\lambda}(\boldsymbol{x}, t)=\sum_{s=1}^{p} a_{s}\left(\boldsymbol{x}-\boldsymbol{x}_{s}\right) \mathcal{G}_{\lambda_{s}}^{\lambda} g^{\lambda_{s}}\left(\boldsymbol{x}_{s}, t\right),
\end{aligned}
$$

where the summation is carried out over the collocation points $\boldsymbol{x}_{s}$ and $a_{s}$ are interpolation functions. Below, we use the third-order Lagrange polynomials. The reconstructions (32) and (33) take into account that the reference frames $\lambda_{s}$ at various collocation points $x_{s}$ may differ from one another. Thus, the corresponding populations $f^{\lambda_{s}}$ and $g^{\lambda_{s}}$ are transformed into a target reference frame $\lambda$ using Eqs. (29) and (30) prior to the interpolation.

The evaluation of the populations at the monitoring point $\boldsymbol{x}$ at time $t$ involves the propagation and the collision steps. In the propagation step, semi-Lagrangian advection is performed using the reconstruction (32) and (33) at the departure points of characteristic lines,

$$
\begin{aligned}
& f_{i}(\boldsymbol{x}, t)=\bar{f}_{i}\left(\boldsymbol{x}-\boldsymbol{v}_{i} \delta t, t-\delta t\right), \\
& g_{i}(\boldsymbol{x}, t)=\bar{g}_{i}\left(\boldsymbol{x}-\boldsymbol{v}_{i} \delta t, t-\delta t\right) .
\end{aligned}
$$

Here, particle's velocities $\boldsymbol{v}_{i}$ are defined relative to the comoving local reference frame at $\boldsymbol{x}$ and $t$. In order to find the co-moving reference frame, a predictor-corrector process is executed as follows: In the predictor step, the local reference frame $\lambda_{0}=\left\{\boldsymbol{u}_{0}, T_{0}\right\}$ is initialized using the local flow velocity and the local temperature, which are available from the previous time step, $\boldsymbol{u}_{0}=\boldsymbol{u}(\boldsymbol{x}, t-\delta t), T_{0}=T(\boldsymbol{x}, t-\delta t)$. The density, momentum and temperature are consequently computed by

$$
\begin{gathered}
\rho_{1}=\sum_{i=0}^{Q-1} f_{i}^{\lambda_{0}}, \\
\rho_{1} \boldsymbol{u}_{1}=\sum_{i=0}^{Q-1} \boldsymbol{v}_{i}^{\lambda_{0}} f_{i}^{\lambda_{0}}, \\
\rho_{1} E_{1}=\sum_{i=0}^{Q-1} \frac{\left(\boldsymbol{v}_{i}^{\lambda_{0}}\right)^{2}}{2} f_{i}^{\lambda_{0}}+\sum_{i=0}^{Q-1} g_{i}^{\lambda_{0}} .
\end{gathered}
$$

The computed velocity (36) and temperature (37) define the corrector reference frame $\lambda_{1}=\left\{\boldsymbol{u}_{1}, T_{1}\right\}$ at the monitoring point and the propagation step (34) is repeated with the updated reference frame. The predictor-corrector process is iterated until convergence with the limit values,

$$
\rho(\boldsymbol{x}, t), \boldsymbol{u}(\boldsymbol{x}, t), T(\boldsymbol{x}, t), f_{i}^{\lambda(\boldsymbol{x}, t)}=\lim _{n \rightarrow \infty} \rho_{n}, \boldsymbol{u}_{n}, T_{n}, f_{i}^{\lambda_{n}},
$$

defining the density, velocity, temperature and the precollision populations at the monitoring point $x$ at time $t$. The predictor-corrector iteration loop ensures that the propagation and the collision steps are performed at the co-moving reference frame, in which the local equilibrium populations (16) and (17) are exact.

\section{Lattice reference frame}

If instead of an adaptive co-moving reference PonD frame, the fixed lattice reference frame $\lambda_{L}=\left\{\mathbf{0}, T_{L}\right\}$ is used, we arrive at the semi-Lagrangian LBM $[9,10,39]$. In this special case, the lattice equilibrium populations $f_{i}^{L}, g_{i}^{L}$ are evaluated by transforming the exact equilibrium populations (16) and (17) to the lattice reference frame,

$$
\begin{gathered}
f^{L}=\mathcal{G}_{\{\boldsymbol{u}, T\}}^{\left\{0, T_{L}\right\}} f^{\mathrm{eq}}, \\
g^{L}=\left(C_{v}-\frac{D}{2}\right) T f^{L} .
\end{gathered}
$$

Similarly, the quasi-equilibria are transformed to the lattice reference frame,

$$
\begin{aligned}
f^{* L} & =\mathcal{G}_{\{\{\boldsymbol{u}, T\}}^{\left\{0, T_{L}\right\}} f^{*}, \\
g^{* L} & =\mathcal{G}_{\{\boldsymbol{u}, T\}}^{\left\{\boldsymbol{0}, T_{L}\right\}} g^{*} .
\end{aligned}
$$

A discussion is in order here. It is well known that the standard $D 2 Q 9$ lattice with the lattice equilibrium does not allow for a compressible model. At the same time, the mere change to the co-moving reference frame readily provides a compressible model with the same number of discrete velocities. In order to understand why the choice of the reference frames matters, we remind that attention should be paid at the higher-order moments that are not included in the transformation. For the $D 2 Q 9$ lattice these are the diagonal elements of the third-order moment and the diagonal elements of the fourth-order moment,

$$
\begin{aligned}
& Q_{\alpha \alpha \alpha}^{\lambda}=\sum_{i=0}^{8}\left(v_{i \alpha}^{\lambda}\right)^{3} f_{i}^{\lambda}, \\
& R_{\alpha \alpha \alpha \alpha}^{\lambda}=\sum_{i=0}^{8}\left(v_{i \alpha}^{\lambda}\right)^{4} f_{i}^{\lambda} .
\end{aligned}
$$

While the off-diagonal third-order elements, $Q_{x y y}=M_{12}$ and $Q_{y x x}=M_{21}$, as well as the off-diagonal fourth-order element $R_{x y}=M_{22}$ are the same in both, the co-moving and the lattice reference frame since they are among the moments transformed, the equilibrium values of the non-transformed moments are not equal in both references. Indeed, using the co-moving equilibrium (16) we find,

$$
Q_{\alpha \alpha \alpha}^{e q}=3 \rho T u_{\alpha}+\rho u_{\alpha}^{3},
$$




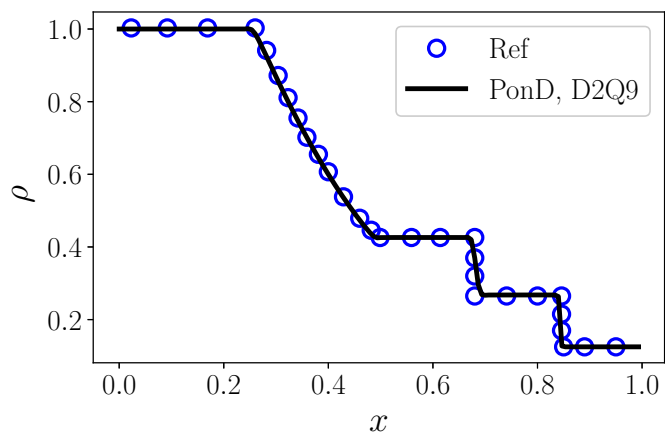

FIG. 1. Density profile for Sod's shock tube, at time $t^{*}=0.2$, with the PonD $D 2 Q 9$ model.

$$
R_{\alpha \alpha \alpha \alpha}^{e q}=3 \rho T^{2}+6 \rho T u_{\alpha}^{2}+\rho u_{\alpha}^{4}
$$

With the diagonal moments (45) and (46), the equilibrium third-order moment tensor and the once-contracted equilibrium fourth-order moment tensor become,

$$
\begin{gathered}
Q_{\alpha \beta \gamma}^{e q}=\rho T\left(u_{\alpha} \delta_{\beta \gamma}+u_{\beta} \delta_{\alpha \gamma}+u_{\gamma} \delta_{\alpha \beta}\right)+\rho u_{\alpha} u_{\beta} u_{\gamma} \\
R_{\alpha \beta}^{e q}=\rho T\left((D+2) T+u^{2}\right) \delta_{\alpha \beta}+\rho\left((D+4) T+u^{2}\right) u_{\alpha} u_{\beta} .
\end{gathered}
$$

The equilibrium Maxwell-Boltzmann relations (47) and (48) are precisely what is required to recover the compressible flow equations in the thermodynamic limit. On the contrary, the lattice equilibrium (41) returns, instead of (45) and (46),

$$
\begin{gathered}
Q_{\alpha \alpha \alpha}^{L}=3 \rho T_{L} u_{\alpha}, \\
R_{\alpha \alpha \alpha \alpha}^{L}=3 \rho T_{L}^{2}+3 \rho T_{L} u_{\alpha}^{2} .
\end{gathered}
$$

Finally, if the temperature is fixed to the lattice temperature $T_{L}$, the athermal model is recovered with

$$
f^{L}=\mathcal{G}_{\left\{\boldsymbol{u}, T_{L}\right\}}^{\left\{\mathbf{0}, T_{L}\right\}} f^{\mathrm{eq}} .
$$

In summary, while the $D 2 Q 9$ velocity set relative to the fixed lattice reference frame is restricted to isothermal and low Mach flows, the same velocity set relative to the co-moving reference frame can capture compressible flows. To demonstrate this point, we show the results for Sod's shock tube, which is a typical benchmark Riemann problem for a compressible flow solver. The initial conditions for the flow field are,

$$
\left(\rho, u_{x}, P\right)= \begin{cases}(1.0,0.0,0.15), & x / L_{x}<0.5 \\ (0.125,0.0,0.015), & x / L_{x}>0.5\end{cases}
$$

where $L_{x}=600$ is the number of grid points. The viscosity is $\mu=0.001$ and $\gamma=1.4$. The results for the density profile at non-dimensional time $t^{*}=t \sqrt{T_{l}} / L_{x}=0.2,\left(T_{l}\right.$ is the temperature on the left half tube), are shown in Fig. 1. It is clear that the $D 2 Q 9$ lattice, using PonD, agrees well with the exact solution.

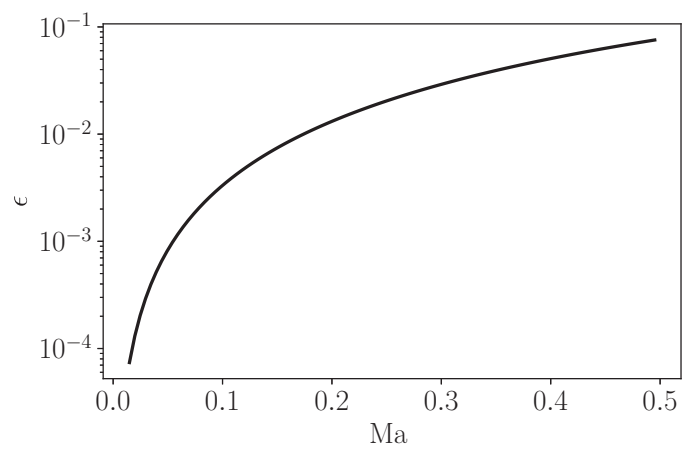

FIG. 2. Deviation of the $x x x$ component of the $D 2 Q 9$ equilibrium third order tensor $Q_{x x x}^{L}$ (49) from its Maxwell-Boltzmann counterpart $Q_{x x x}^{\mathrm{eq}}$ (47) as a function of Mach number $\mathrm{Ma}=u / c_{s} . \epsilon=\left(Q_{x x x}^{L}-\right.$ $\left.Q_{x x x}^{\mathrm{eq}}\right) / Q_{x x x}^{\mathrm{eq}}$. The deviation vanishes for higher-order lattices.

\section{MULTI-SCALE COUPLING SCHEME}

\section{A. The overlap and velocity space refinement}

Each discrete velocity set can be characterized by its domain of validity. Going by an example, in the standard lattice reference frame, the $D 2 Q 9$ lattice provides a reliable simulation of a nearly-incompressible flow as long as the magnitude of the flow velocity stays below $u \lesssim 0.1$ in lattice units. This estimate is based on the deviation of the diagonal element of the equilibrium third-order moment [Eq. (49)] from the Maxwell-Boltzmann value [Eq. (47)] (see Fig. 2). The nextorder velocity sets in the Hermite hierarchy of Table I provide a larger domain of validity [5]. It is therefore expected that the use of a higher-order velocity set instead of the $D 2 Q 9$ can improve the accuracy of the simulation in certain cases. At the same time, using the higher-order velocity set in the entire computational domain all the time is demanding and wasteful whenever the accuracy of the lower-order model is sufficient. Since the semi-Lagrangian propagation is not bound to a specific lattice and while both the higher- and the lower-order models can be realized on the same grid, we seek an adaptive realization where a higher-order model is used only if the flow situation demands more accuracy than it is expected from the lower-order model while the lower-order model is used whenever its accuracy suffices.

Let us consider two velocity sets of different order,

$$
\begin{aligned}
& \mathcal{V}_{q}=\left\{\boldsymbol{v}_{i}^{q}, i=0, \ldots, q-1\right\}, \\
& \mathcal{V}_{Q}=\left\{\boldsymbol{v}_{i}^{Q}, i=0, \ldots, Q-1\right\},
\end{aligned}
$$

where $q<Q$. At a monitoring point $\boldsymbol{x}$, at time $t$, we can choose between the two corresponding population vectors, $f_{q}$ and $f_{Q}$. We further assume that at $(\boldsymbol{x}, t)$ the validity domains of the higher-order $Q$-model and the lower-order $q$-model overlap and both models are equally applicable. Switching between the models requires one of the two operations:

(i) The lifting operation switches from the lower-order $q$ model to the higher-order $Q$-model and is required to increase the accuracy if the available state $f_{q}$ is close to the limit of its validity domain and we need to proceed with the higher-order model. The lifting operation thus requires to specify a map,

$$
f_{q} \rightarrow f_{Q}
$$


(ii) The projection operation switches from the higherorder $Q$-model to the lower-order $q$-model and allows to maintain the accuracy with lesser velocities whenever the state $f_{Q}$ can be replaced by a lower-order state $f_{q}$ within the validity domain of the lower-order model. The projection thus requires to specify a map,

$$
f_{Q} \rightarrow f_{q}
$$

Note that the lifting and the projection operations are applied when the lower- and the higher-order models are both valid and a proper transfer of information between the different models is possible, i.e., their validity domains must overlap. This is a general requirement that the models of any multi-scale method must respect. We shall now specify the two switching operations separately.

\section{B. Lifting}

We use a notation $m_{q}$ for the $q$-dimensional vector of moments of the low-order model, available at $(\boldsymbol{x}, t)$. With a $q \times q$ matrix $\mathcal{M}_{q}$, we have,

$$
m_{q}=\mathcal{M}_{q} f_{q}
$$

On the other hand, a moment vector of the $Q$-populations $M_{Q}$ can be considered as an element of a direct sum,

$$
M_{Q}=M_{q} \oplus M_{Q-q},
$$

where $M_{q} \in \operatorname{Im}\left(\mathcal{M}_{q}\right)$ is a vector from the image of the matrix $\mathcal{M}_{q}$ while $M_{Q-q}$ is the rest of the higher-order moments. The lifting operation consists in specifying the respective contributions as,

$$
\begin{gathered}
M_{q}=m_{q}, \\
M_{Q-q}=M_{Q-q}^{\mathrm{eq}} .
\end{gathered}
$$

This construction can be understood as a decomposition between the locally conserved, the "slow" and the "fast" components. The information of the low-order population vector are fully retained, both equilibrium and non-equilibrium contributions, and the moments $M_{q}$ are identified as the slow components. On the other hand, the missing moments $M_{Q-q}$ are considered as fast moments, which are strongly enslaved by the dynamics of the slow moments. The lifted moment vector $M_{q \rightarrow Q}$ is thus written as

$$
M_{q \rightarrow Q}=m_{q} \oplus M_{Q-q}^{\mathrm{eq}} .
$$

With the moments $M_{q \rightarrow Q}$ completed, the lifted populations $f_{Q}$ are found by moment inversion,

$$
f_{Q}=\mathcal{M}_{Q}{ }^{-1} M_{q \rightarrow Q},
$$

where $\mathcal{M}_{Q}$ is the $Q \times Q$ moments-to-populations transform for the higher-order model.

\section{Projection}

In the projection step, a high-order population vector $f_{Q}$ is mapped to a lower-order $f_{q}$. In this case, the high-order lattice represents the $Q$ linearly independent moments, including the subset of the first $q$ moments, which is required to construct the populations $f_{q}$ of the low-order lattice. Hence, in contrast

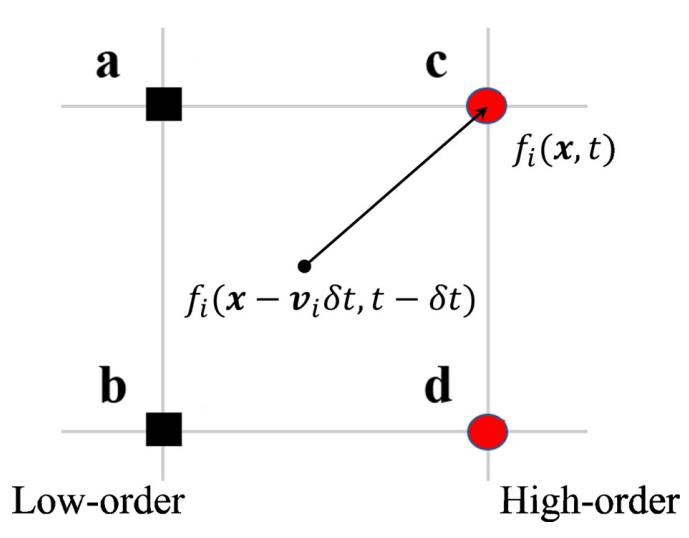

FIG. 3. Schematic of the multi-scale semi-Lagrangian propagation. For illustration purposes we use four collocation points for the interpolation and we consider the case of lifting operation. We monitor the propagation step of the high-order population $f_{i}(\boldsymbol{x}, t)$ (point $\boldsymbol{c}$ ). The departure point $\boldsymbol{x}_{\mathrm{dep}, i}=\boldsymbol{x}-\boldsymbol{v}_{i} \delta t$ (black circle) is surrounded by the collocation points $\boldsymbol{a}$ to $\boldsymbol{d}$. The lifting operation is applied at the points $\boldsymbol{a}$ and $\boldsymbol{b}$, which use the low-order velocity set. The semi-Lagrangian step concludes with the interpolation of the populations at the departure point.

to the lifting procedure, there is no missing information and all linearly independent moments $m_{Q \rightarrow q}$ are operationally available from $f_{Q}$,

$$
m_{Q \rightarrow q}=M_{q},
$$

and the population vector $f_{q}$ is obtained by moment inversion,

$$
f_{q}=\mathcal{M}_{q}{ }^{-1} m_{Q \rightarrow q}
$$

The concepts of the two mappings are generic and apply to both the $f$-populations (density and momentum lattice) and $g$-populations (energy lattice) described above. Finally, we point out that both the lifting and the projection are local operations, which leads to high efficiency, numerical stability and flexibility for an adaptive velocity refinement.

\section{Semi-Lagrangian propagation}

We now discuss combination of the lifting and projection operations with the semi-Lagrangian propagation. To that end, let us consider a population $f_{i}(\boldsymbol{x}, t)$, corresponding to a discrete velocity $\boldsymbol{v}_{i}$ of either the lower- or the higher-order velocity set. The semi-Lagrangian propagation starts with the calculation of the departure point $\boldsymbol{x}_{\mathrm{dep}, i}=\boldsymbol{x}-\boldsymbol{v}_{i} \delta t$, and with identifying the surrounding collocation points [cf. Eq. (32)]. Here we must consider that, in general, the collocation points utilize velocity sets of different orders. Thus, the lifting and the projection operations should be applied such that the populations involved in the reconstruction are all expressed in the same velocity set (see a schematic in Fig. 3). The implementation of the semi-Lagrangian propagation can be summarized in the following steps, (see Appendix $\mathrm{C}$ for a pseudo-code listing of the algorithm):

(1) Calculate the departure point, $\boldsymbol{x}_{\mathrm{dep}, i}=\boldsymbol{x}-\boldsymbol{v}_{i} \delta t$ and identify the collocation points.

(2) For all collocation points:

(a) If velocity set order is lower than the velocity set order of $(\boldsymbol{x}, t)$, apply lifting. 
(b) If velocity set order is higher than velocity set order of $(\boldsymbol{x}, t)$, apply projection.

(3) Calculate $f_{i}\left(\boldsymbol{x}_{\mathrm{dep}, i}, t-\delta t\right)$ using the reconstruction formula [Eq. (32)].

The first and third steps of the semi-Lagrangian propagation are the same as for the case of a single velocity set throughout the domain, whereas the second step is only carried out near the interface of different velocity sets. The above multi-scale modification of the semi-Lagrangian algorithm is independent of the underlying model and can be used with single or double populations and with static or co-moving reference frames (PonD). In the latter case, the semi-Lagrangian propagation is performed iteratively in a predictor-corrector loop. During the iterations, the lifting/projection operations need to be performed once to each point that is close to the interface region. The conventional collision step follows the propagation, according to the specified model.

\section{E. Adaptive refinement of velocity space}

The multi-scale scheme is concluded by adaptive refinement of the velocity sets at all collocation points, according to the underlying refinement criterion. The specification of the refinement criterion amounts to choosing an appropriate flow variable (Mach or Knudsen numbers) and a corresponding threshold, above which the high-order velocity set is to be used. The choice of the threshold value has been discussed in Sec. III A and its effect on the accuracy is also assessed numerically in the test cases below. Note that despite the use of a fixed number as a threshold, the multi-scale approach is indeed fully adaptive, in the sense that the monitoring flow variable is computed at each grid point and the velocity set is refined where it is needed, according to the local characteristics of the flow. In this work, Mach and Knudsen number serve as monitoring flow variables for the refinement criterion. We note however that this is not unique and other variables can be used if the nature of the flow under study demands so.

Before proceeding with the validation of the proposed scheme, a comment on the multi-scale problems is in order. Multi-scale flows are characterized by a substantial variation of characteristic quantities which most commonly include but are not limited to spatial scales, time scales, Mach number or Knudsen number. In what follows, we only consider the Mach or the Knudsen number variation. For the spatio-temporal scales variation, we refer to existing grid refinement techniques [43]. However, when devising a numerical scheme, which bridges such large variations by coupling different methods for different scales, it is important to realize that consistent coupling is only possible if there is a region, where the validity range of both schemes overlap. In our case, this corresponds to regions of the flow, where both lattices can provide an accurate description of the flow field. These regions will eventually become the interface region between both lattices and thus correspond to the velocity space refinement. Hence, if such overlap regions do not exist in the flow, the use of a multi-scale scheme may become inappropriate.

\section{NUMERICAL RESULTS AND DISCUSSION}

\section{A. Multi-scale flows}

In this section, the performance and accuracy of the multiscale scheme is probed using the velocity sets generated by the Gauss-Hermite quadrature, as described in Sec. II A. The time step $\delta t$ in all simulations below is such that the Courant-Friedrichs-Lewy (CFL) number is given by $\mathrm{CFL}=$ $\max \left|c_{i \alpha}\right|(\delta t / \delta x)=1.0$, where $c_{i \alpha}$ are Hermite roots in Table I. After assessing the convergence of the proposed coupling of different velocity sets in a static arrangement, using the twodimensional Taylor-Green vortex setup in Sec. IV A 1, we shall probe the adaptive coupling with an athermal advected vortex in Sec. IV A 2 and an athermal jet flow in Sec. IV A 3, using a fixed reference frame $\lambda=\left\{\boldsymbol{0}, T_{L}\right\}$. The underlying model for these simulations uses a single $f$-lattice, without quasi-equilibrium relaxation. The motivation for using different velocity sets in these cases stems from the errors of the standard $D 2 Q 9$ lattice as the flow velocity increases, and the refinement criterion between the higher- and lower-order velocity sets is based on a Mach number threshold. Finally, we consider the shock structure problem in Sec. IV A 4 using a co-moving reference frame (PonD) and the full compressible model ( $f$ - and $g$-populations with variable Prandtl number and adiabatic exponent), in which the choice of the velocity set is dictated by the variation of the Knudsen number.

\section{Taylor-Green vortex}

The multi-scale scheme is first validated with an incompressible, low-Mach Taylor-Green vortex, using the $D 2 Q 9$ and the $D 2 Q 25$ lattices. The reference solution is,

$$
\begin{gathered}
u_{x}(x, y, t)=-u_{0} \cos (k x) \sin (k y) e^{-2 v k^{2} t}, \\
u_{y}(x, y, t)=u_{0} \sin (k x) \cos (k y) e^{-2 v k^{2} t}, \\
p(x, y, t)=p_{0}-\frac{\rho u_{0}^{2}}{4}(\cos (2 k x)+\cos (2 k y)) e^{-4 v k^{2} t},
\end{gathered}
$$

where $k=2 \pi / L$ is the wave number while $u_{0}$ and $p_{0}$ are the initial values of velocity and pressure, respectively. A $128 \times 128$ grid was used with periodic boundary conditions. The velocity $u_{0}$ is set such that the initial maximum Mach number is $\mathrm{Ma}=u_{0} / \sqrt{T_{L}}=0.05$ and the Reynolds number is set to $\operatorname{Re}=u_{0} L / v=50$. Under these flow conditions, both the low- and the high-order lattices are expected to be equally valid and therefore the case is suitable for an initial validation of the consistency and convergence properties of the coupling scheme. The higher-order $D 2 Q 25$ model is used inside a circle, with radius $L / 3$, centered in the domain and the $D 2 Q 9$ lattice is used outside the circle, as shown in Fig. 4. The velocity sets for this case are defined from the outset and maintained throughout the simulation. The results of the simulation agree well with the reference solution. Figure 5(a) shows the $y$ component of the flow velocity across the horizontal line passing though the center of the domain is compared with the reference solution for times $t_{1}^{*}=0.057, t_{2}^{*}=0.57, t_{3}^{*}=$ 1.15 , where $t^{*}=t\left(2 \nu k^{2}\right)$. Finally, a convergence study of the multi-scale scheme is shown in Fig. 5(b). For the convergence study the $L_{2}$ error norm based on the $x$-velocity is used. The results show that second-order accuracy is maintained and the 


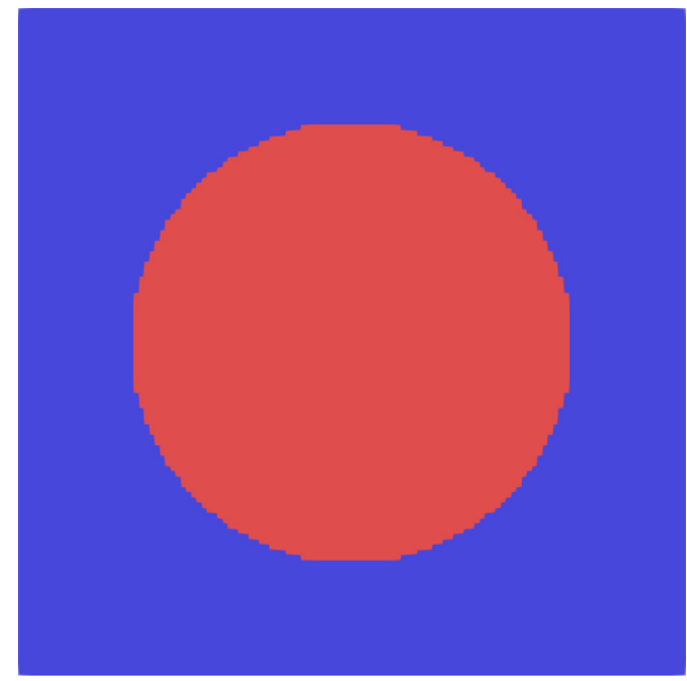

Velocity set

- D2Q9 - D2Q25

FIG. 4. Velocity set distribution for the Taylor-Green vortex.

proposed coupling scheme does not degrade or interfere with the numerical properties of the individual velocity sets.

\section{Athermal vortex advection}

The advection of an athermal vortex is studied next with the proposed multi-scale scheme, using the $D 2 Q 9$ and the $D 2 Q 25$ lattices. The initial conditions for the velocity and the density are,

$$
\begin{gathered}
u_{x}=U_{0}-\epsilon\left(\frac{y-y_{c}}{R_{c}}\right) \exp \left[-\frac{\left(x-x_{c}\right)^{2}+\left(y-y_{c}\right)^{2}}{2 R_{c}^{2}}\right], \\
u_{y}=\epsilon\left(\frac{x-x_{c}}{R_{c}}\right) \exp \left[-\frac{\left(x-x_{c}\right)^{2}+\left(y-y_{c}\right)^{2}}{2 R_{c}^{2}}\right], \\
\rho=\rho_{0} \exp \left[-\frac{M a_{v}^{2}}{2} \exp \left(-\frac{r^{2}}{R_{c}^{2}}\right)\right],
\end{gathered}
$$

where $U_{0}$ is the advection velocity, $\epsilon$ is the strength of the vortex and $R_{c}$ is its characteristic radius [44]. The advection and vortex Mach numbers are set to $\mathrm{Ma}_{\mathrm{a}}=0.25$ and $\mathrm{Ma}_{\mathrm{v}}=$ 0.6 , respectively. A $200 \times 200$ grid was used with periodic boundary conditions and the viscosity is set to $\mu=0.01$. A threshold Mach number $\mathrm{Ma}_{\text {thr }}$ for switching between the $D 2 Q 9$ and $D 2 Q 25$ velocity sets can be inferred from the discussion in Secs. II C 2 and III A: In the lattice reference frame, the accuracy of the $D 2 Q 9$ model is limited by the deviation of the equilibrium third-order moment [Eq. (49)] from its Maxwell-Boltzmann value [Eq. (45)] (see Fig. 2). In the present example, we set a threshold value $\mathrm{Ma}_{\mathrm{thr}}=0.3$, which allows for a maximum $3 \%$ deviation when the $D 2 Q 9$ model is used.

Figure 6 shows the distribution of the two velocity sets, the density and the local Mach number at two different times. The higher-order $D 2 Q 25$ velocity set is wrapped around the high-speed flow region and adapts to the advection of the
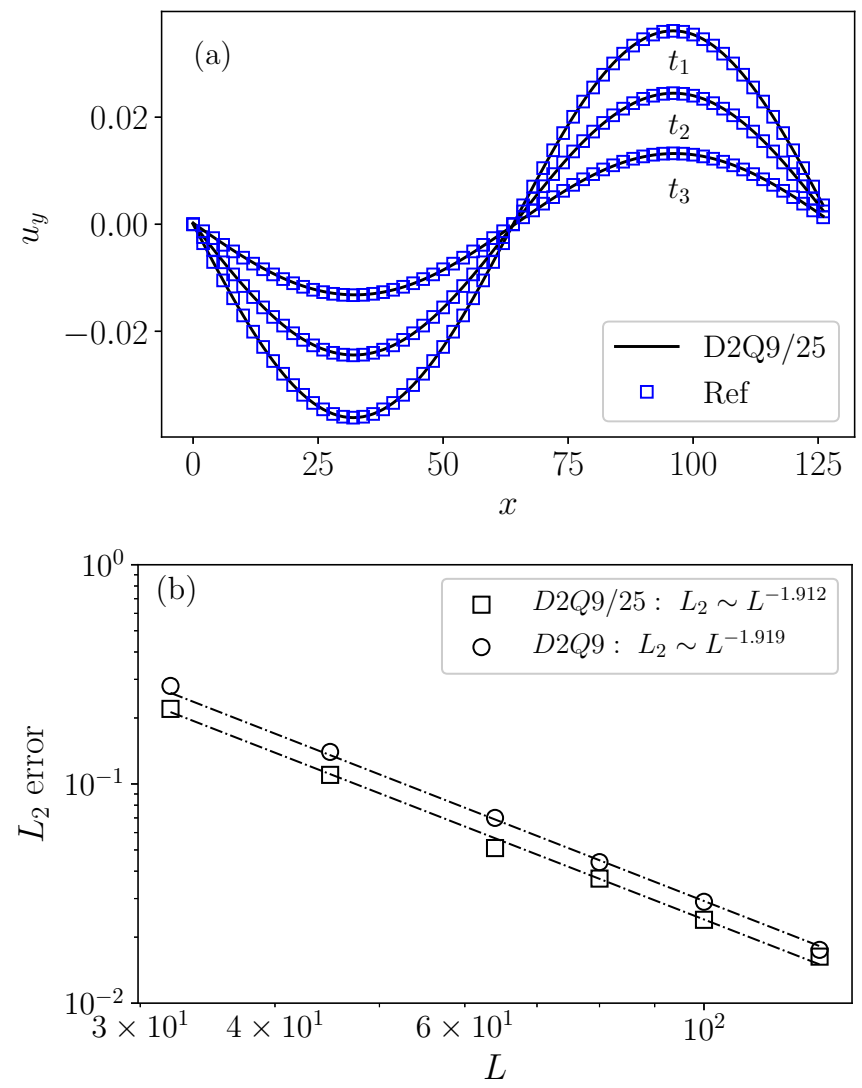

FIG. 5. (a) Comparison of the $y$-velocity with the analytical solution (64) at three times, $t_{1}^{*}=0.057, t_{2}^{*}=0.57$ and $t_{3}^{*}=1.15$. (b) Scaling of $L_{2}$ error for the $D 2 Q 9$ and the coupled $D 2 Q 9 / 25$ models. The $L_{2}$ error is computed based on $x$-velocity.

vortex. The coupling scheme was compared with the simulation in which a single velocity set is used throughout the domain. In Fig. 7, density contours are shown for the $D 2 Q 9$, the $D 2 Q 25$ and the hybrid $D 2 Q 9 / 25$ models, at $t=500$. The insufficient isotropy of the $D 2 Q 9$ velocity set manifests in the distortion of the vortex, in contrast with the cases of both the $D 2 Q 25$ and the coupled $D 2 Q 9 / 25$ models. Figure 8 shows the density profiles across the center of the vortex for simulations using a single velocity set $(D 2 Q 9$ and $D 2 Q 25)$ and for the hybrid velocity set $D 2 Q 9 / 25$, for three different Mach number thresholds, $\mathrm{Ma}_{\mathrm{thr}}=(0.27,0.3,0.4)$. Convergence of the hybrid $D 2 Q 9 / 25$ is observed, with the decrease of the Mach number threshold. This is expected since the Mach number threshold represents the accuracy of the equilibrium moments of the low-order velocity set.

\section{Athermal jet flow}

In a two-dimensional jet setup, the inflow velocity profile consists of a low-speed base flow with a Mach number $\mathrm{Ma}_{1}=0.15$ and a high-speed region, which is superimposed in the center of the domain with diameter $D_{\text {jet }}$ and Mach number $\mathrm{Ma}_{\mathrm{h}}=0.6$. At the inlet, a zero pressure gradient and fixed velocity are imposed, whereas a non-reflecting boundary condition is prescribed at the outlet. Free-stream boundary conditions are applied at the top and bottom planes. The 
(a)

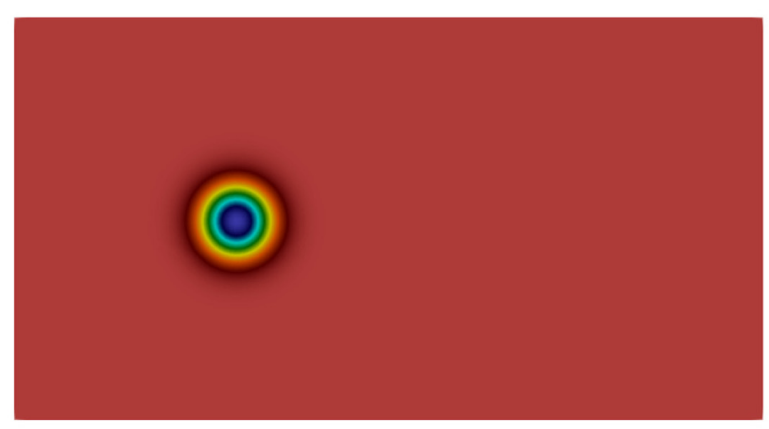

(c)

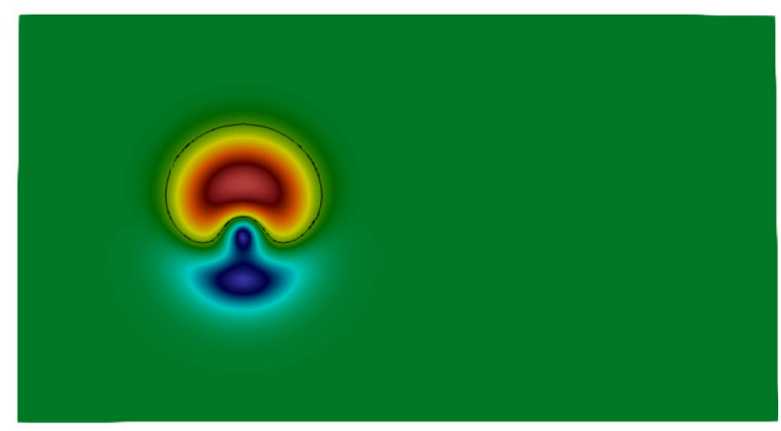

Mach

(e)

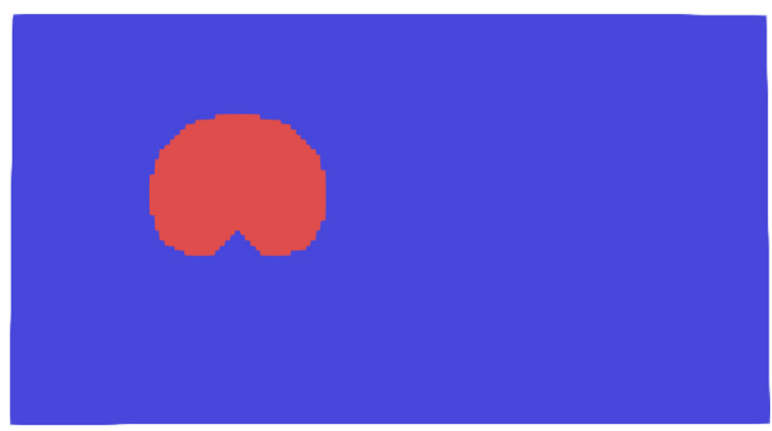

Velocity set

D2Q9 - D2Q25 (b)
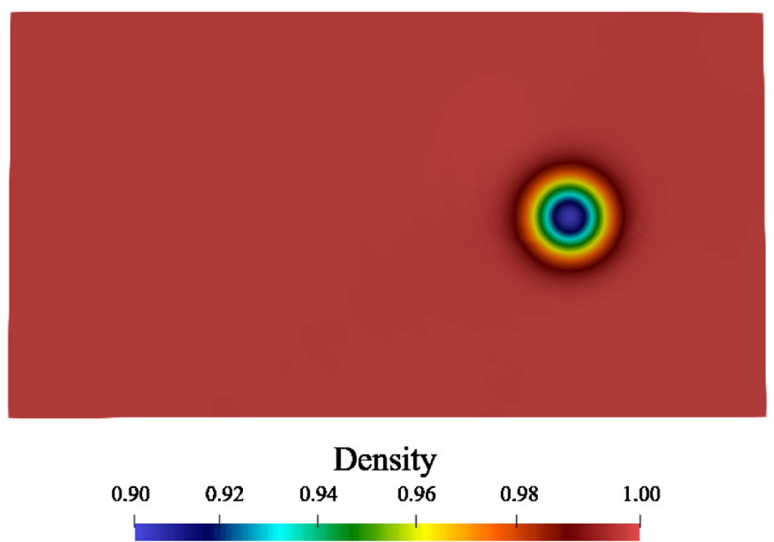

(d)
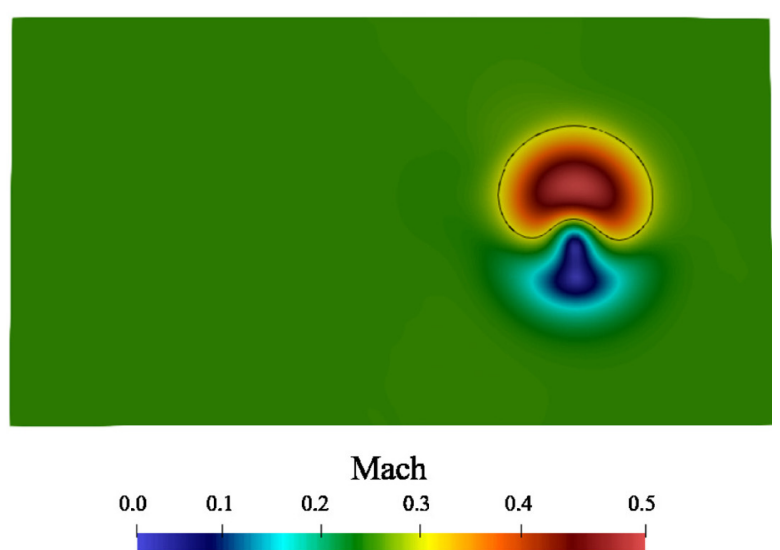

(f)

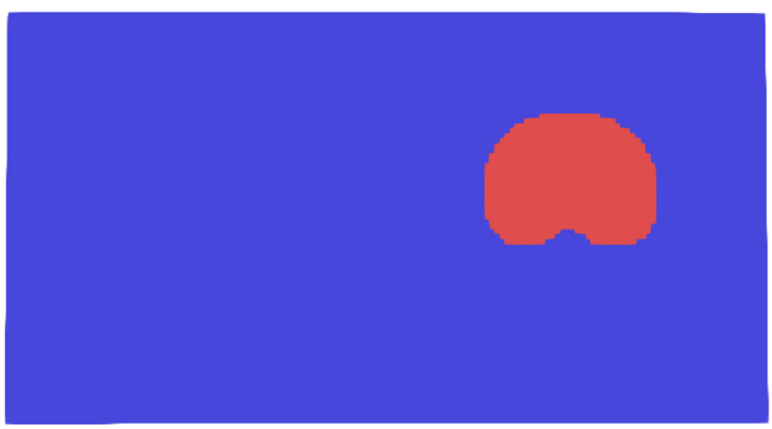

Velocity set

D2Q9 $\square$ D2Q25

FIG. 6. Vortex advection with the coupled $D 2 Q 9 / 25$ athermal model. (a), (b): Density; (c), (d): Mach number; (e), (f): Velocity set. Snapshots at $t_{1}=0$ (left) and $t_{2}=500$ (right). Black contour in (b), (c) corresponds to the threshold Mach number Mathr $=0.3$. The higherorder $D 2 Q 25$ patch follows the advected vortex.

simulation is carried out on a $750 \times 500$ grid with a jet diameter $D_{\text {jet }}=25$ and the Reynolds number is set to $\operatorname{Re}=$ $D_{\text {jet }} U_{\text {jet }} / v=10^{3}$, where $U_{\text {jet }}=\operatorname{Ma}_{\mathrm{h}} \sqrt{T_{L}}$.

Same as in the example of Sec. IV A 2, the adaptive multiscale model was based on the $D 2 Q 9$ and $D 2 Q 25$ velocity sets, in the lattice reference frame. The refinement criterion was based on the local Mach number and was set to $\mathrm{Ma}_{\mathrm{thr}}=0.3$. Figure 9 shows the instantaneous $x$-velocity field and the corresponding spatial distribution of the discrete velocity sets. The time average results for the velocity set and the $x$-velocity are shown in Fig. 10, where it is evident that the $D 2 Q 25$ is dominant across the centerline and near the jet inlet whereas the $D 2 Q 9$ is used at low-speed regions of the flow. Note that the average ratio of the nodes using the higher-order velocity set is about $\sim 15 \%$ of the total number of nodes. A further discussion of the computational speed-up is presented in Sec. IV C. In Fig. 11, the multi-scale simulation using the hybrid $D 2 Q 9 / 25$ lattice is further compared with the simulations in which the $D 2 Q 9$ and $D 2 Q 25$ were applied uniformly in the entire domain. Figure 11 shows the average $x$-velocity (normalized with the maximum velocity of the jet) across the center-line, and the average $x$-velocity at two normal sections at distances $x_{c} / D_{\text {jet }}=12,16$ from the inlet. It is apparent that the multi-scale simulation is in good agreement with the simulation using the $D 2 Q 25$ model throughout. In a contrast, the $D 2 Q 9$ model shows significant deviations with respect to the 


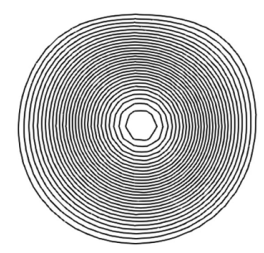

D2Q9

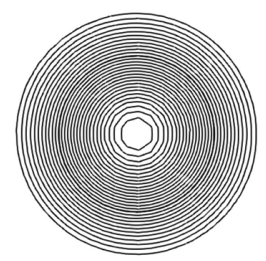

D2Q25

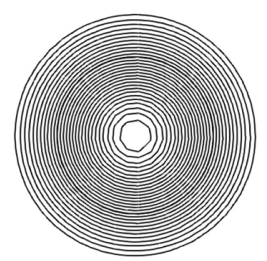

D2Q9/25
FIG. 7. Density contours of an athermal advected vortex simulation with $D 2 Q 9, D 2 Q 25$ and the coupled $D 2 Q 9 / 25$.

$D 2 Q 25$ model. This further validates the proposed multi-scale scheme.

\section{Shock structure}

The shock structure problem is a classical problem in kinetic theory of gases, in which non-equilibrium effects dominate the flow [45]. It is well known that since the thermodynamic variables vary on a scale of a few mean free paths, the continuum Navier-Stokes-Fourier equations fail to correctly describe the shock wave structure. We consider a quasi one-dimensional plane shock wave, with an initial step of density, velocity and temperature, at the center of the computational domain. The upstream and downstream flow values are connected through the Rankine-Hugoniot conditions [46]. The upstream mean free path for hard sphere molecules is defined as,

$$
\lambda_{1}=\frac{16}{5 \sqrt{2 \pi \gamma}}\left(\frac{\mu_{1} \alpha_{1}}{p_{1}}\right),
$$

where $p_{1}, \alpha_{1}, \mu_{1}$ are the pressure, the speed of sound and the viscosity of the gas upstream of the shock, respectively. The viscosity varies with the temperature as,

$$
\mu=\mu_{1}\left(\frac{T}{T_{1}}\right)^{s},
$$

where for the case of hard spheres $s=0.5$. The steady-state non-dimensional density, temperature, normal stress and heat

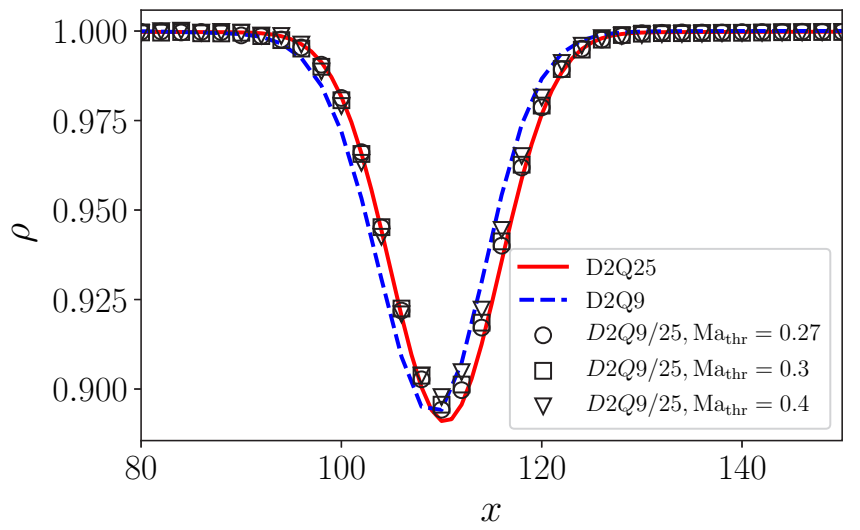

FIG. 8. Density of an athermal advected vortex simulation with $D 2 Q 9, D 2 Q 25$ and coupled $D 2 Q 9 / 25$. The simulations for the coupled $D 2 Q 9 / 25$ have been performed with three different Mach number thresholds, $\mathrm{Ma}_{\mathrm{thr}}=(0.27,0.3,0.4)$. (a)

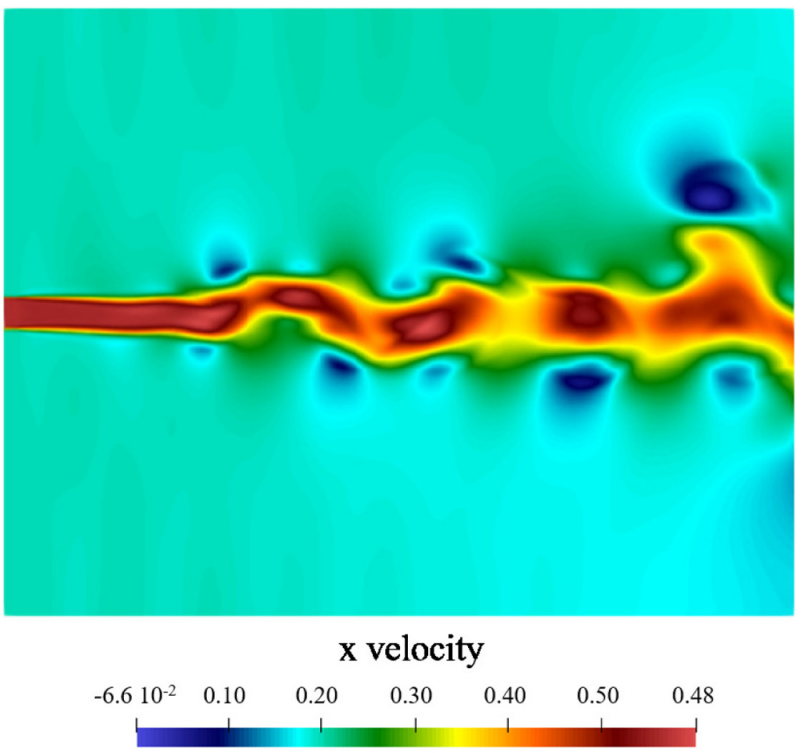

(b)

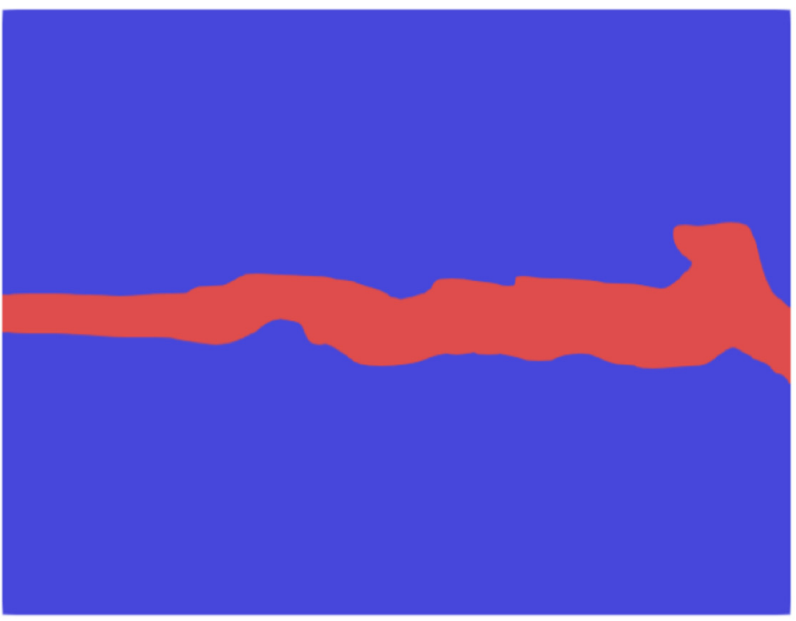

Velocity Set

D2Q9 D2Q25

FIG. 9. Simulation of athermal jet flow with the coupled $D 2 Q 9 / 25$ model. (Top) Snapshot of the $x$-velocity field; (Bottom) velocity set throughout the domain.

flux are defined as follows,

$$
\begin{array}{ll}
\rho_{n} & =\frac{\rho-\rho_{1}}{\rho_{2}-\rho_{1}}, \quad T_{n}=\frac{T-T_{1}}{T_{2}-T_{1}}, \quad \hat{\sigma}_{x x}=\frac{\sigma_{x x}}{p_{1}}, \\
\hat{q}_{x} & =\frac{q_{x}}{p_{1} \sqrt{2 T_{1}}},
\end{array}
$$

where the subscripts 1 and 2 indicate the upstream and downstream values, respectively.

We use the two-population PonD method of Sec. II B, with Prandtl number $\operatorname{Pr}=2 / 3$ and adiabatic exponent of monoatomic ideal gas $\gamma=5 / 3$. The model was realized with the coupling of the $D 2 Q 16$ and the $D 2 Q 25$ velocity sets, with the higher-order $D 2 Q 25$ used inside the region of the shock wave. The refinement criterion was based on the density-based 
(a)
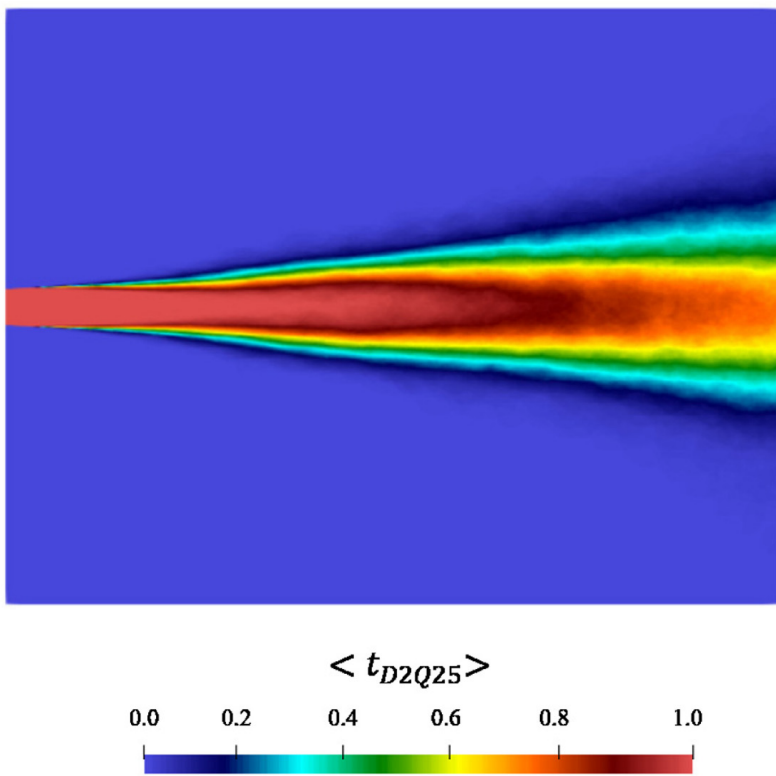

(b)

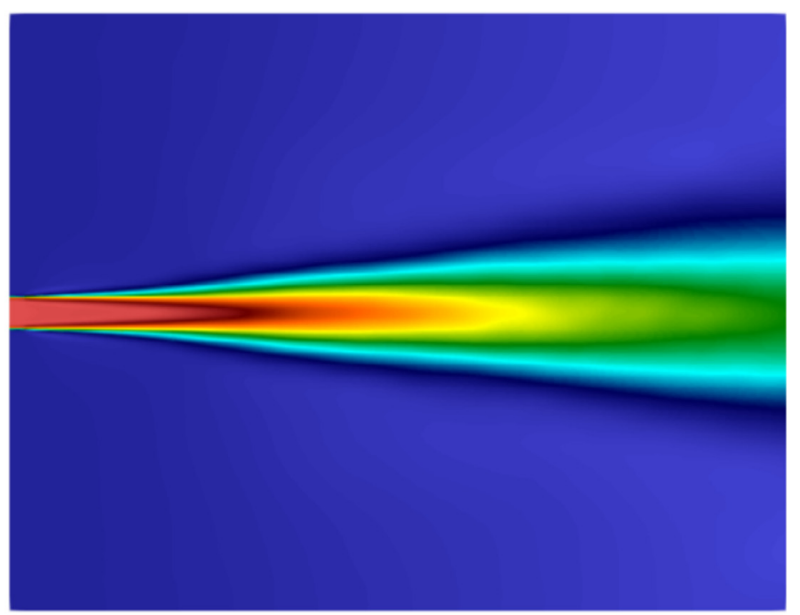

X velocity

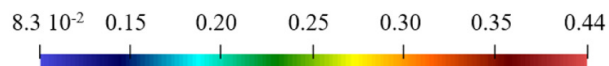

FIG. 10. Simulation of athermal jet flow with the coupled $D 2 Q 9 / 25$ model. (Top) Fraction of time using the higher-order $D 2 Q 25$ velocity set; (Bottom) Time average of the $x$-velocity.

local Knudsen number,

$$
\mathrm{Kn}=\frac{\lambda}{\left|\frac{\rho}{d \rho / d x}\right|} .
$$

The threshold value was set to $\mathrm{Kn}_{\text {thr }}=0.02$, a typical value suggested in the literature [38,47]. The simulations were carried out with a quasi one-dimensional setup, with grid resolution $\delta x=0.02 \lambda_{1}$. The velocity set in the domain is initialized with the $D 2 Q 16$ lattice and wherever the local Knudsen number exceeds the threshold value is refined to $D 2 Q 25$. The time evolution of the velocity set and the computed Knudsen number are shown in Fig. 12. The $D 2 Q 25$ is initially concentrated in the center of the domain and gradually expands towards the entire shock layer. The steady-state
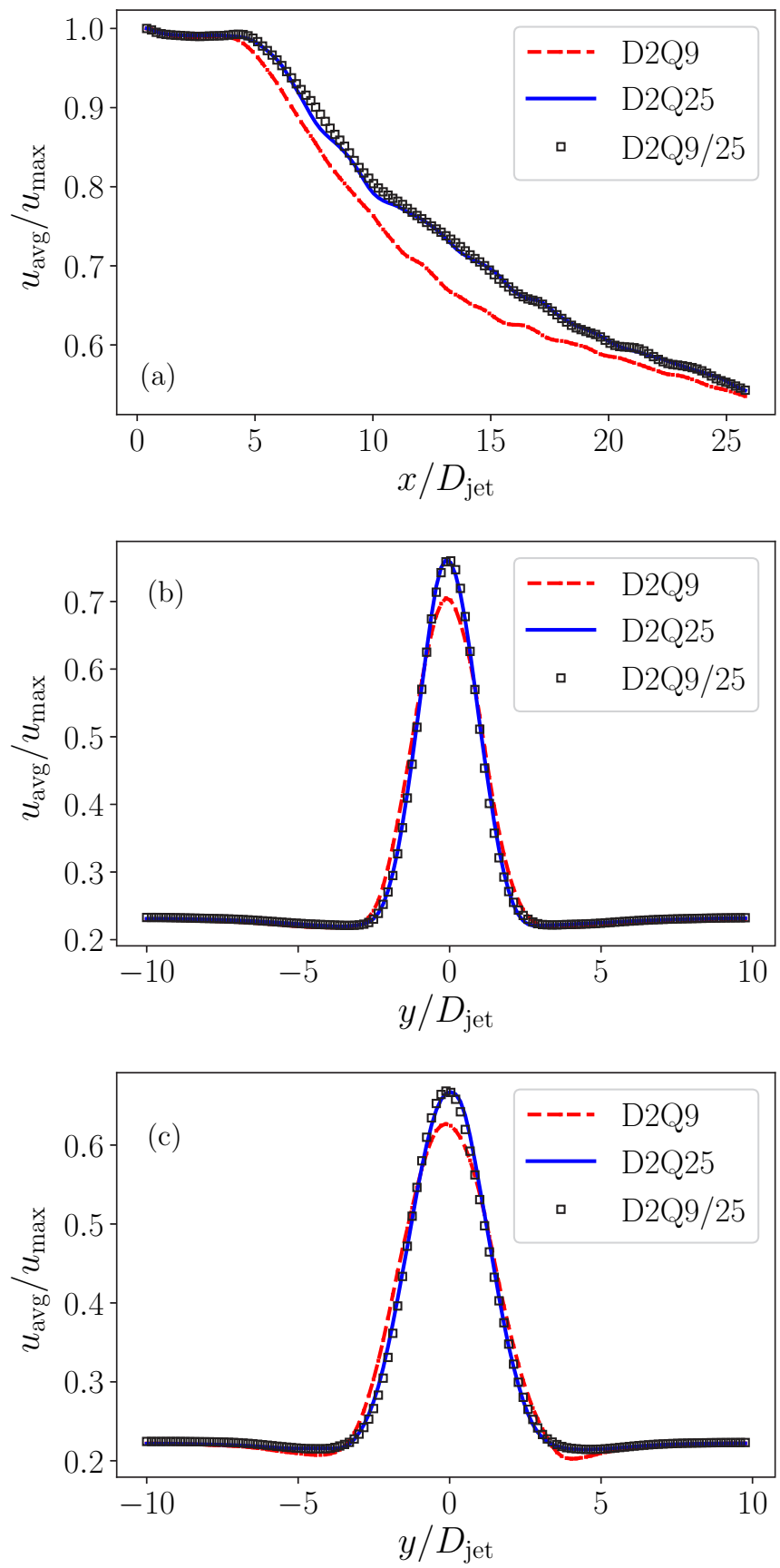

FIG. 11. Comparison of athermal jet flow with $D 2 Q 9, D 2 Q 25$ and coupled $D 2 Q 9 / 25$. (a) Mean streamwise velocity profiles along the centerline; (b) Mean streamwise velocity profiles at $x / D_{\text {jet }}=12$; (c) Mean streamwise velocity profiles at $x / D_{\text {jet }}=16$.

results for $\mathrm{Ma}=1.6$ are shown in Fig. 13 and compared with the results of Ohwada [48]. The origin of the coordinate system is the point with $\rho_{n}=0.5$ and $x_{n}=x / 0.5 \sqrt{\pi} \lambda_{1}$ is used as the reduced coordinate. It can be seen that the density, temperature and velocity profiles agree well with the reference data. The normal stress and heat flux profiles are also in good agreement with the reference data. Finally, we demonstrate the effect of the Knudsen number threshold on the steadystate results. Two simulations with $\mathrm{Kn}_{\mathrm{thr}}=(0.02,0.05)$ are compared in Fig. 14. As expected, the domain occupied by 

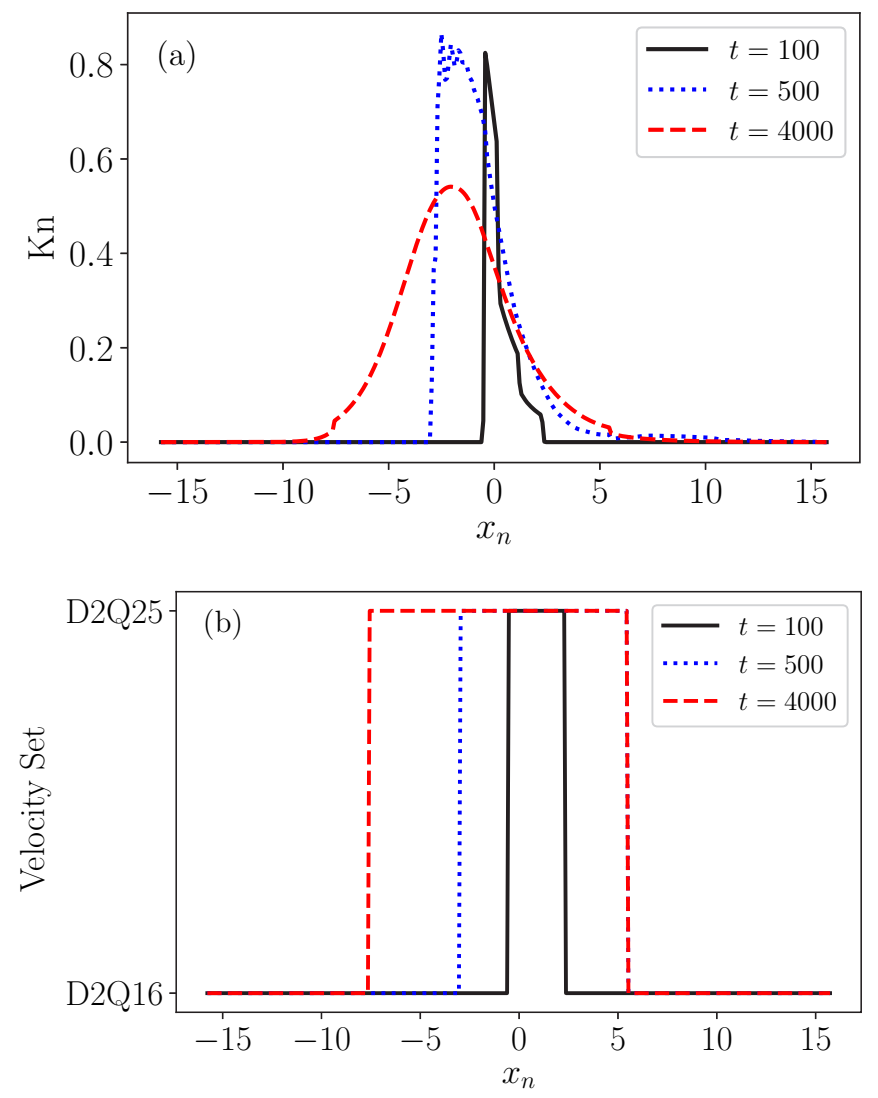

FIG. 12. Evolution of Knudsen profile (top) and velocity set (bottom) at times $t=100,500,4000$.

the high-order velocity set at steady-state shrinks with the increase of the Knudsen number threshold. Additionally, it is observed that the profiles of the flow variables, obtained with the two different thresholds, agree well with each other. However, small deviations occur near the interface region of the different velocity sets, for the case of the higher Knudsen number threshold $\left(\mathrm{Kn}_{\mathrm{thr}}=0.05\right)$.

\section{B. Micro-Couette flow}

The simulation of microscale flows is of both practical and theoretical interest. A classical example is the shear driven Couette flow. It is well known that both the choice of the discrete velocity set and the boundary conditions have a strong impact on the accuracy at a non-vanishing Knudsen number. In particular, the standard $D 2 Q 9$ lattice does not suffice to capture the Knudsen layer, resulting in a significant deviation of the velocity profile. Furthermore, it was reported that even-orders velocity sets perform significantly better than odd-orders [27].

Note that the micro-Couette flow is not a true multi-scale problem. Indeed, the Knudsen number is uniform in the entire domain, in contrast to the shock-structure problem, where the Knudsen number varies within the shock layer. For a quantitative discussion, we use the analytical solution of the micro-Couette flow for the $D 2 Q 9$ and $D 2 Q 16$ lattices [11]. While the $D 2 Q 9$ model can predict a slip-flow solution, it fails in the transient regime at $\mathrm{Kn} \gtrsim 0.1$. The $D 2 Q 16$ model, on
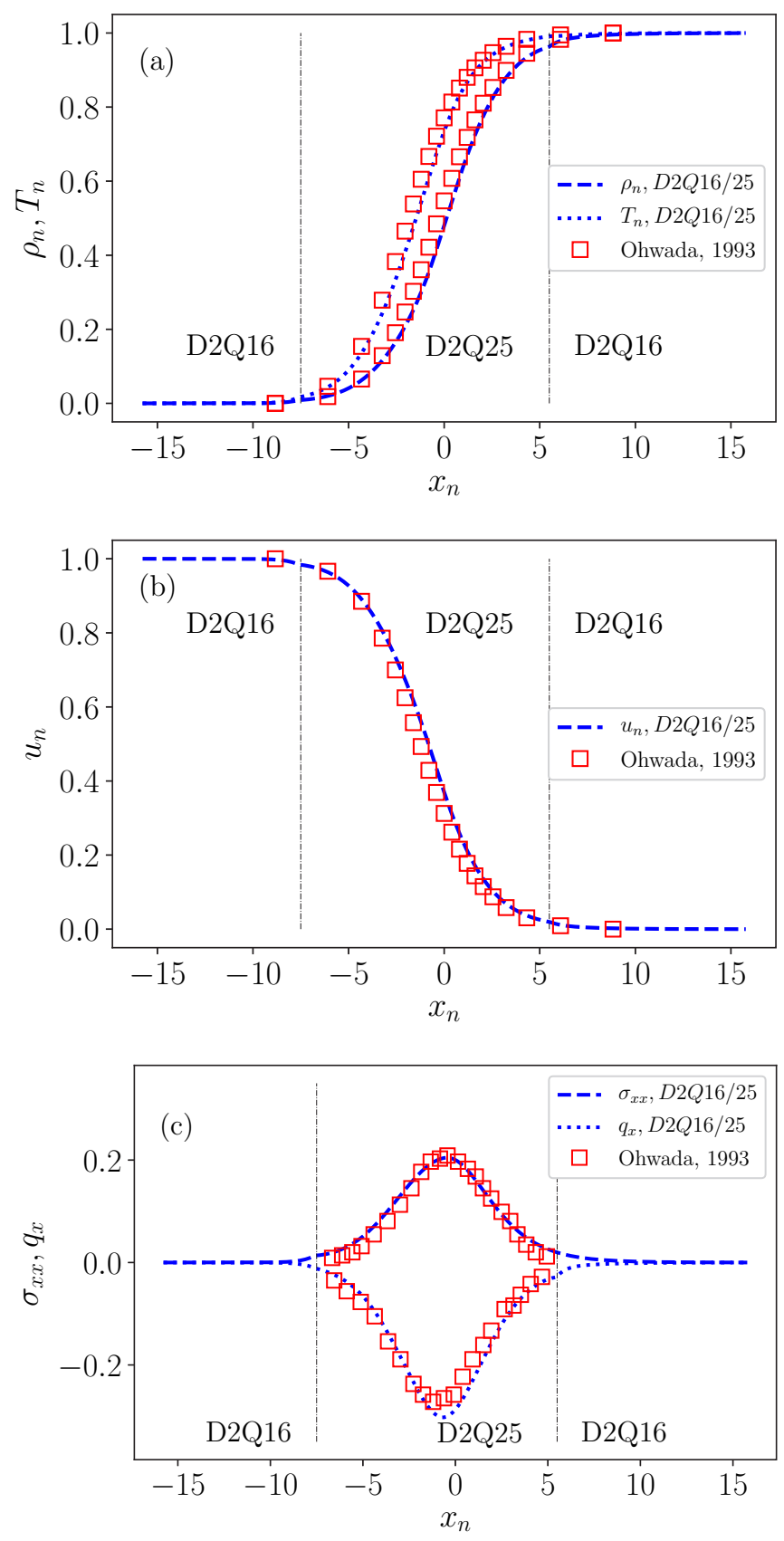

FIG. 13. Multi-scale $D 2 Q 16 / 25$, shock-structure for $\mathrm{Ma}=1.6$. Density, temperature, velocity, normal stress and heat flux profiles, compared with results of Ohwada [47].

the other hand, improves the accuracy of the solution considerably and predicts the boundary Knudsen layer in qualitative agreement with kinetic theory [45]. Figure 15 shows the relative error $\epsilon=\left|u_{9}-u_{16}\right| /\left|u_{16}\right|$, where $u_{9}, u_{16}$ are the velocity profiles from the analytical solutions, obtained by $D 2 Q 9$ and $D 2 Q 16$ models respectively, for Knudsen numbers $\mathrm{Kn}=0.1$ and $\mathrm{Kn}=0.2$. At a constant Knudsen number, the relative error has a constant value in the main flow and grows in the vicinity of the wall boundaries. Increasing the Knudsen number leads to magnification of the error in the main flow as well as extending the influence of the wall due to the boundary Knudsen layer. 

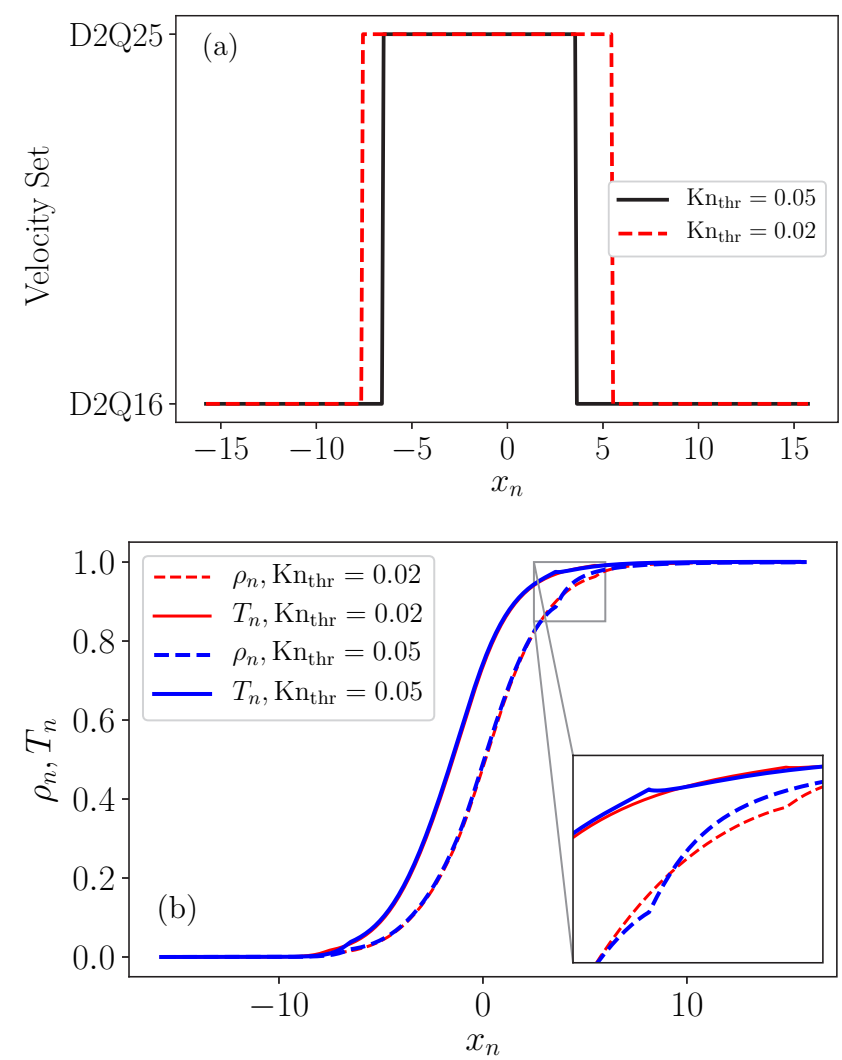

FIG. 14. Multi-scale $D 2 Q 16 / 25$, shock-structure for $\mathrm{Ma}=1.6$. Velocity set (top), density and temperature profiles (bottom) for two thresholds of Knudsen number, $\mathrm{Kn}_{\mathrm{thr}}=(0.02,0.05)$.

The setup of the Couette test case consists of two plates surrounding the fluid, which move with opposite velocities $u_{w}= \pm 0.1$ and same temperature $T_{w}=T_{L}$. The simulations were performed with a quasi-one dimensional $300 \times 1$ grid. We test the multi-scale scheme with the D2Q9 velocity set in the main flow and the D2Q16 near the wall boundaries. The underlying model consists of the two-population realization of the PonD method, with Prandtl number fixed to $\operatorname{Pr}=2 / 3$ and adiabatic exponent of monoatomic ideal gas $\gamma=5 / 3$. Diffusive boundary conditions were implemented $[27,49]$. For

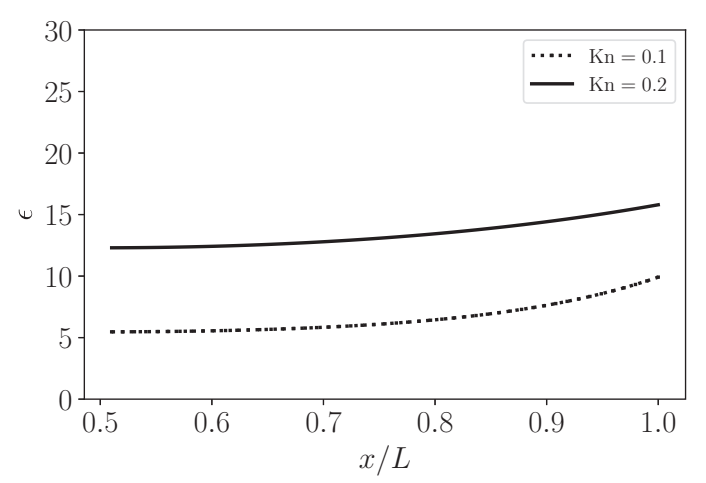

FIG. 15. Relative error $\epsilon=\left|u_{9}-u_{16}\right| /\left|u_{16}\right|$ between the $D 2 Q 9$ and $D 2 Q 16$ models for the Couette flow, where $u_{9}$ and $u_{16}$ are the analytical velocity profiles [11] for the $D 2 Q 9$ and the $D 2 Q 16$ models, respectively. the Couette flow setup, the Knudsen number is defined as [27],

$$
\mathrm{Kn}=\frac{\mu \sqrt{T}}{p L},
$$

where $p$ and $L$ denote the pressure and the distance between sharing plates, respectively. The results for the nondimensional velocity (normalized with the difference of the wall velocities) for Knudsen numbers $\mathrm{Kn}=0.1, \mathrm{Kn}=0.2$ and $\mathrm{Kn}=0.4$ are presented in Fig. 16, in which comparison is made with results from linearized Bhatnagar-Gross-Krook (BGK) equation [27]. The dotted vertical lines on the plots indicate the $D 2 Q 9 / 16$ interface for the multi-scale simulations. The position of the $D 2 Q 9 / 16$ interface is set according to the theoretical error analysis (1\% above the main flow error).

We observe that for all Knudsen numbers the multi-scale scheme matches well with $D 2 Q 9$ solution in the main flow and also with the reference results near the wall boundaries, due to the local use of the $D 2 Q 16$ lattice. However, a small discontinuity develops near the interface region of the different velocity sets, which becomes more prominent with increasing Knudsen number. The source of this discontinuity is that with increasing Knudsen number, the validity domains of the low- and the high-order models do not overlap at the interface region (in other words, they are not equally valid), as suggested by the relative error in Fig. 15.

\section{Computational efficiency}

In this section, we investigate the increase of computational efficiency when using the multi-scale scheme. To that end, a computational domain of the size $N \times N$ (shown in Fig. 17) is decomposed in two rectangular domains which use the high-order $D 2 Q 25$ and low-order $D 2 Q 9$ velocity sets, respectively. The ratio of the CPU times $t_{\mathrm{D} 2 \mathrm{Q} 25} / t_{\mathrm{hyb}}$, where $t_{\mathrm{D} 2 \mathrm{Q} 25}$ refers to the time of a globally used $D 2 Q 25$ and $t_{\text {hyb }}$ to the hybrid scheme, is measured as a function of the domain size $N$, keeping the ratio of the high-order nodes to $10 \%$ of the computational domain fixed. The measurements were carried out using the Taylor-Green setup on an eight-core PC without parallelization (Intel Core i7-9700@3GHz) and the timings are shown in Fig. 18. The speedup that can be observed for the simulation using the $D 2 Q 9$ lattice in the entire domain is $t_{D 2 Q 25} / t_{D 2 Q 9}=2.9$. Thus, the maximum speedup that can be achieved when the ratio of $D 2 Q 25$ is fixed to $10 \%$ is $\left(t_{D 2 Q 25} / t_{\text {hyb }}\right)_{\max }=2.43$ (as shown below). For small domain sizes, the computational cost due to the lifting and projection operations at the interface nodes limits the speedup but for larger domains $(N>100)$ a significant speedup is observed, saturating close to the maximum value. The proposed multiscale scheme can therefore lead to significant savings in CPU time.

We proceed with further analysis of the computational cost of the multi-scale scheme. The number of operations involved in both lifting and projection can be estimated as summarized in Tables II and III. For the lifting operation, we start with a given population vector $f_{q}$. We first need to calculate the highorder equilibrium $f_{Q}^{\text {eq }}$. The equilibrium can be constructed as $f_{Q}^{\text {eq }}=\rho \mathcal{M}_{Q} W_{Q}$, where $\mathcal{M}_{Q}$ is a $Q \times Q$ matrix depending on flow velocity and $W_{Q}$ is the vector of weights (step 1 , Table II). The next step is to evaluate the first $q$ moments of $f_{q}$ (step 

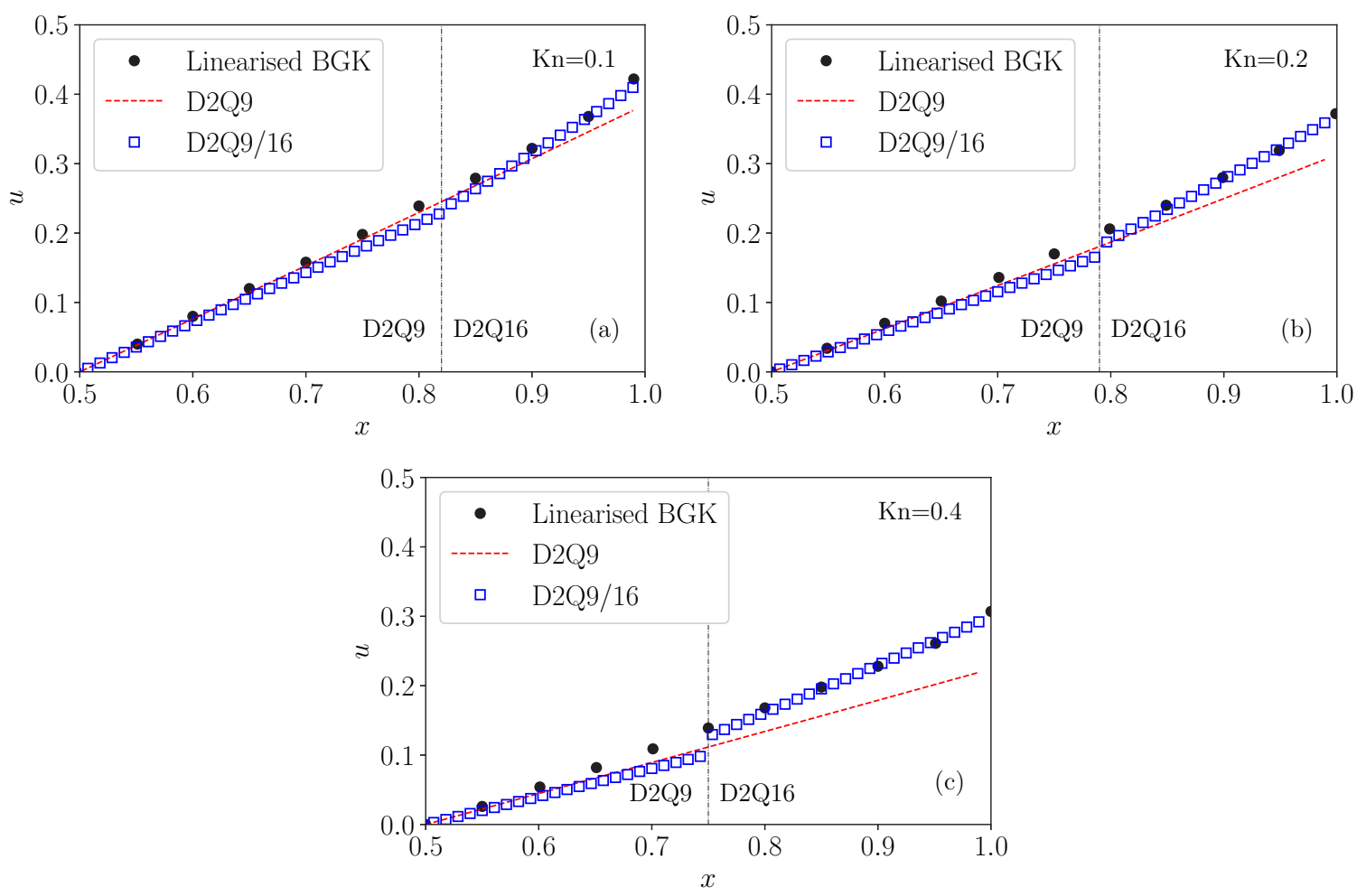

FIG. 16. Shear driven Couette flow with $D 2 Q 9, D 2 Q 16$ and coupled $D 2 Q 9 / 16$. Profiles of the normalized velocity for Knudsen numbers $\mathrm{Kn}=0.1$ (top); $\mathrm{Kn}=0.2$ (middle); $\mathrm{Kn}=0.4$ (bottom).

2, Table II). The remaining $Q-q$ moments are evaluated from the high-order equilibrium population (step 3, Table II). Finally the vector of $Q$ moments is inverted to population vector (step 4, Table II). Summing up the contributions, we estimate the total number of operations for the lifting as $I_{L} \approx$ $8 Q^{2}+2 q^{2}-2 Q q$. Similarly we can estimate the operations for the projection, which contains much fewer calculations. For this case, we start with a given population vector $f_{Q}$ and we need to evaluate the first $q$ moments (step 1, Table III). Then the moment vector is inverted (step 2, Table III). The total cost for the projection is $I_{P} \approx 2 Q q+2 q^{2}$. For the case of the $D 2 Q 9$ and $D 2 Q 25$ coupling ( $q=9, Q=25)$, we thus can

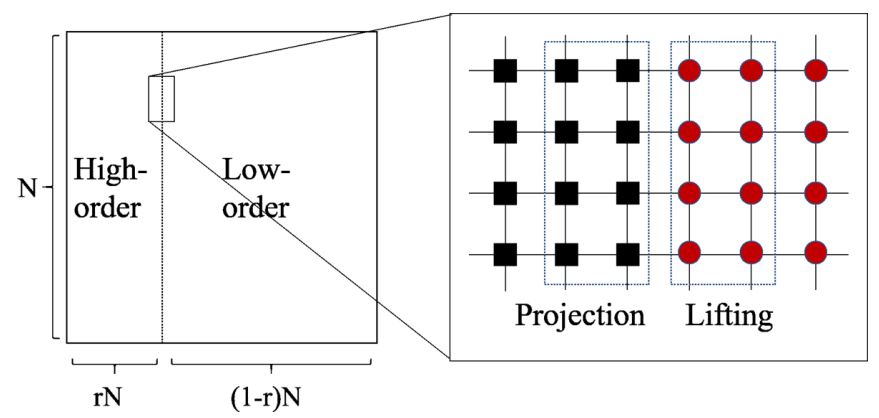

FIG. 17. Schematic for the computational efficiency study. Computational domain of the size $N \times N$ is decomposed into a highand low-order regions. Here $r$ corresponds to the ratio of high-order nodes. For the nodes at the coupling interface (inside dashed boxes), the projection and lifting operations are applied. estimate the ratio between the cost of lifting and projection as $I_{L} / I_{P} \approx 7.5$.

Using the above operation count, we can further evaluate the results of the example considered in Sec. IV C in order to extract the actual cost of the lifting and projection operations relative to the cost of a single step (propagation and collision). We can model the speedup as a function of the domain size $N$ as follows: The time for the globally used $D 2 Q 25$ is $t_{D 2 Q 25}=t_{25} N^{2}$, where $t_{D 2 Q 25}$ is the total CPU time, and $t_{25}$

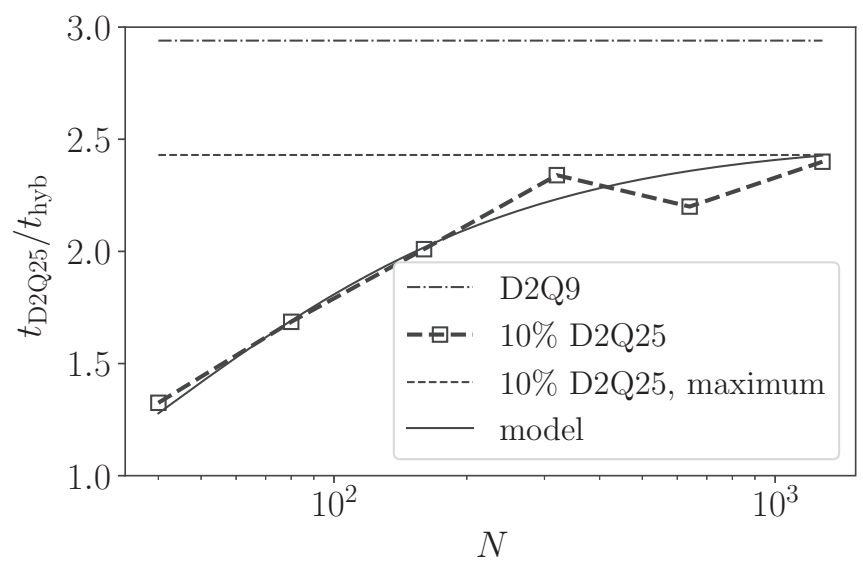

FIG. 18. Speed up ratio $t_{D 2 Q 25} / t_{\text {hyb }}$ as a function of the domain size $N$. Ratio of high-order nodes $D 2 Q 25$ is fixed to $10 \%$. The dashed line corresponds to the theoretical maximum speedup, using $10 \% D 2 Q 25$. The solid line represents the fitted model. 
TABLE II. Operation count for lifting operation.

\begin{tabular}{lcc}
\hline \hline Step & Formula & Operations \\
\hline $\begin{array}{l}\text { 1. Evaluation of high-order } \\
\text { equilibrium }\end{array}$ & $f_{Q}^{\mathrm{eq}}=\rho \mathcal{M}_{Q} W_{Q}$ & $4 Q^{2}$ \\
$\begin{array}{l}\text { 2. Evaluation of the first } q \\
\text { moments }\end{array}$ & $M_{q}=m_{q}$ & $2 q^{2}$ \\
$\begin{array}{l}\text { 3. Evaluation of the missing } \\
Q-q \text { moments }\end{array}$ & $M_{Q-q}=M_{Q-q}^{e q}$ & $2(Q-q) Q$ \\
$\begin{array}{l}\text { 4. Moments inversion to } \\
\text { populations }\end{array}$ & $f_{Q}=\mathcal{M}_{Q}^{-1}\left(M_{q} \oplus M_{Q-q}\right)$ & $2 Q^{2}$ \\
\hline \hline
\end{tabular}

is the CPU time per lattice node. For the hybrid simulation $D 2 Q 9 / 25$, where a fraction $r$ of the domain is covered by the higher-order lattice (see Fig. 17), the total CPU time is,

$$
t_{\text {hyb }}=r N^{2} t_{25}+(1-r) N^{2} t_{9}+\alpha\left(t_{L}+t_{P}\right) N,
$$

where $t_{9}$ is the CPU time per lattice node for the $D 2 Q 9$ lattice and $t_{L}$ and $t_{P}$ are the CPU time for each lifting and projection operation, respectively. Furthermore, $\alpha$ represents the number of grid points that need to be lifted/projected near the interface region and depends on the interpolation stencil. Combining these estimates, the speedup ratio as function of the domain size $N$ becomes,

$$
\frac{t_{D 2 Q 25}}{t_{\mathrm{hyb}}}=\frac{t_{25} N^{2}}{r N^{2} t_{25}+(1-r) N^{2} t_{9}+\alpha\left(t_{L}+t_{P}\right) N} .
$$

At first, we observe that for a sufficiently large domain, the contribution of the projection/lifting to the speedup can be neglected,

$$
\lim _{N \rightarrow \infty} \frac{t_{D 2 Q 25}}{t_{\mathrm{hyb}}}=\frac{t_{25}}{r t_{25}+(1-r) t_{9}} .
$$

For example, at $r=0.1$ and $t_{25} / t_{9}=2.9$ (from timing of the simulations) the maximum speedup ratio is $\left(t_{D 2 Q 25} / t_{\mathrm{hyb}}\right)_{\max }=$ 2.43 , which agrees with the recorded speedup from the simulations. To estimate the ratio $t_{P} / t_{25}$, we fit the model to the observed speedup using $t_{L} / t_{P} \approx 7.5$ and $\alpha=2$ for the thirdorder Lagrange interpolation. The fitting of the model to the data is shown in Fig. 17 , determining the ratio as $t_{P} / t_{25} \approx 1.0$.

\section{CONCLUSION}

In this work, we presented a multi-scale scheme with an adaptive velocity set, according to a refinement criterion based on the local Mach or Knudsen number. Velocity sets of different order are coupled through the lifting and projection operators. Both operations involve only local computations, which results in a robust and flexible adaptive velocity refinement. The multi-scale scheme can be implemented with either a static or co-moving reference frame and with different models (single/double population, quasi-equilibrium

TABLE III. Operation count for projection operation.

\begin{tabular}{lcc}
\hline \hline Step & Formula & Operations \\
\hline 1. Evaluation of the first $q$ moments & $m_{Q \rightarrow q}=M_{q}$ & $2 q Q$ \\
2. Moments inversion to populations & $f_{q}=\mathcal{M}_{q}^{-1} m_{Q \rightarrow q}$ & $2 q^{2}$ \\
\hline \hline
\end{tabular}

models). The numerical results of a variety of flows demonstrate both accuracy and efficiency of the proposed scheme. This work focused on $2 D$ applications, but the lifting and projection, underlying the coupling scheme, hold also in three dimensions. Investigation of the proposed scheme in three dimensions is left for future work.

\section{ACKNOWLEDGMENTS}

This work was supported by European Research Council (ERC) Advanced Grant No. 834763-PonD. Computational resources at the Swiss National Super Computing Center CSCS were provided under Grants No. s1066, and No. s897.

\section{APPENDIX A: PonD TRANSFORMATION MATRIX}

The implementation of the PonD involves the transformation of a population vector from one reference frame $\lambda=$ $\{\boldsymbol{u}, T\}$ to another reference frame $\lambda^{\prime}=\left\{\boldsymbol{u}^{\prime}, T^{\prime}\right\}$. The transformation is a linear operation, based on the moments matching,

$$
f^{\lambda^{\prime}}=\mathcal{M}_{\lambda^{\prime}}^{-1} \mathcal{M}_{\lambda} f^{\lambda}=\mathcal{G}_{\lambda}^{\lambda^{\prime}} f^{\lambda}
$$

Below, we provide a simple script to obtain analytical expression for the transformation matrix $\mathcal{G}_{\lambda}^{\lambda^{\prime}}$, for the case of $D 2 Q 9$ velocity set, using the symbolic toolbox of Matlab. The script can used for other velocity sets, with a redefinition of the discrete velocities and of the corresponding list of moments.

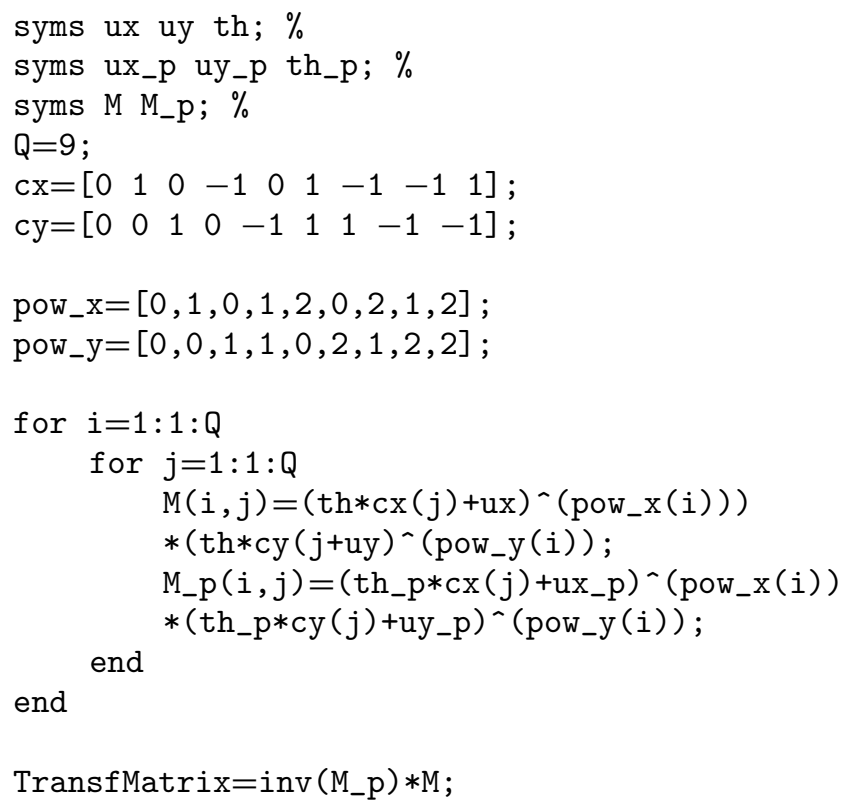

\section{APPENDIX B: LIFTING AND PROJECTION OPERATIONS}

To illustrate the coupling scheme, we consider the lifting and projection operations for the case of the $D 2 Q 9 / D 2 Q 16$ coupling. The velocity set of the $D 2 Q 9$ lattice, $\left\{\boldsymbol{v}_{i}^{9}\right\}$, is formed by the tensor product of the discrete velocities in $1 D$, $(-c, 0, c)$, while the $D 2 Q 16$ lattice, $\left\{\boldsymbol{v}_{i}^{16}\right\}$, is formed by the tensor product of $\left(-c_{2},-c_{1}, c_{1}, c_{2}\right)$. 
For the lifting operation, we need to construct the higherorder moment vector $M_{9 \rightarrow 16}=\left[M_{00}, M_{10}, \cdots, M_{33}\right]$, given the lower-order population vector $f$. The nine first moments are directly computed from $f$,

$$
M_{k l}=\sum_{i=0}^{i=8}\left(v_{i x}^{9}\right)^{k}\left(v_{i y}^{9}\right)^{l} f_{i},
$$

where $k, l \in[0,2]$. The remaining moments are computed from the higher-order equilibrium population $f^{\text {eq, } 16}$,

$$
M_{k l}=\sum_{i=0}^{i=15}\left(v_{i x}^{16}\right)^{k}\left(v_{i y}^{16}\right)^{l} f_{i}^{\mathrm{eq}, 16} .
$$

Finally, the inverse of the moment matrix $\mathcal{M}_{16}^{-1}$ is applied to $M_{9 \rightarrow 16}$,

$$
f_{9 \rightarrow 16}=\mathcal{M}_{16}^{-1} M_{9 \rightarrow 16} .
$$

For the projection operation we compute the moment vector $M_{16 \rightarrow 9}=\left[M_{00}, M_{10}, \cdots, M_{22}\right]$, given the higher-order population vector $f$ as,

$$
M_{k l}=\sum_{i=0}^{i=15}\left(v_{i x}^{16}\right)^{k}\left(v_{i y}^{16}\right)^{l} f_{i},
$$

where $k, l \in[0,2]$. The inverse of the moment matrix $\mathcal{M}_{9}^{-1}$ is applied to $M_{16 \rightarrow 9}$,

$$
f_{16 \rightarrow 9}=\mathcal{M}_{9}^{-1} M_{16 \rightarrow 9} .
$$

The analytical forms of the matrices $\mathcal{M}_{9}^{-1}$ and $\mathcal{M}_{16}^{-1}$ can be obtained from the following MATLAB script,

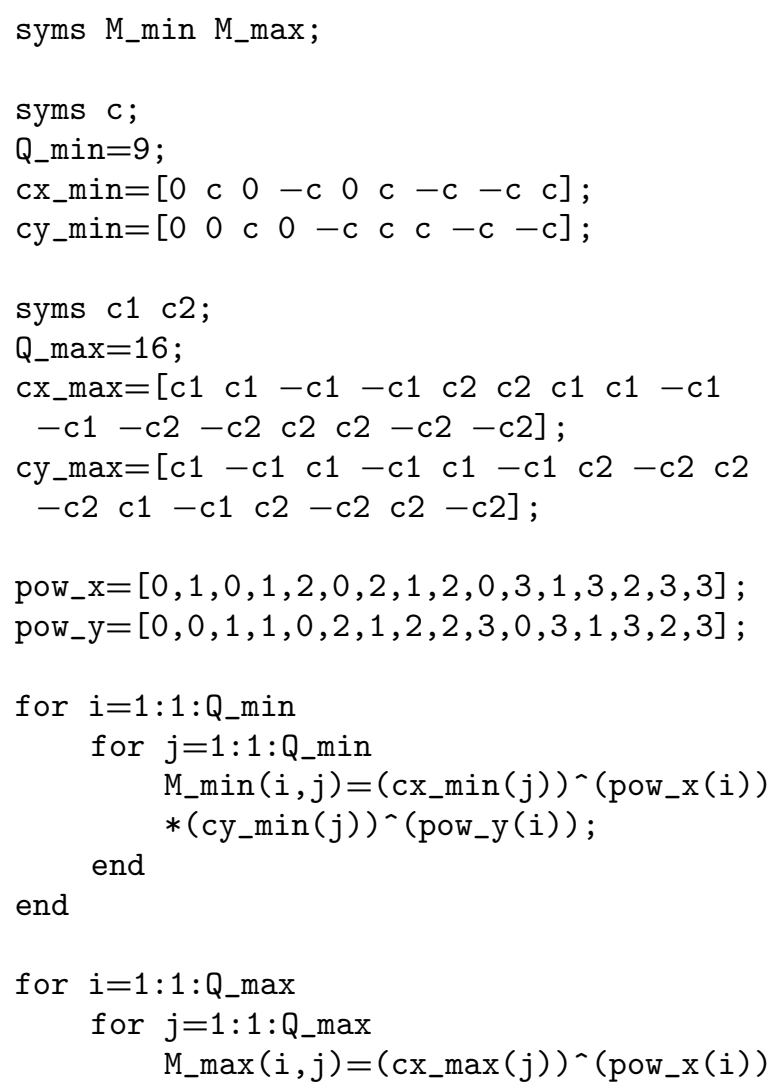

end $*\left(c_{-} y_{\max }(j)\right)^{\wedge}\left(\right.$ pow_y $\left._{-}(i)\right)$;

end

M_min_inv=inv (M_min);

M_max_inv=inv (M_max);

\section{APPENDIX C: PSEUDOCODE LISTING OF MULTI-SCALE SCHEME}

The following pseudocode listing highlights the steps of the semi-Lagrangian propagation and the adaptive refinement of velocity space, according to the proposed multiscale scheme.

//1. Semi-Lagrangian propagation

for node in total_nodes:

for pop in node.populations:

//Calculate departure point

$\mathrm{x}_{-} \mathrm{dep}=$ node. $\mathrm{x}-\mathrm{dt} *$ node. $\mathrm{vx}(\mathrm{pop})$

$y_{-}$dep $=$node. $y-d t *$ node. $v y(p o p)$

//Get collocation points

col_points=collocation_points (x_dep,y_dep)

//Apply lifting or projection where necessary

for col_point in col_points:

if $c o l$ point.vel_set () $<$ node.vel_set(): col_point.lifting ()

if col_point.vel_set() > node.vel_set(): col_point.projection()

//Interpolate to get the population at departure point

f_departure=interpolate (col_points, $x_{-}$dep, y_dep)

.

//2. Collision

...

//3. Update velocity set

//Mach number as a refinement criterion for node in total_nodes:

//Local Mach number

$\operatorname{mach}=$ node $\cdot \operatorname{mach}()$

if mach $>$ mach_thr and node.

vel_set ()$=$ =vset_min :

//node changes to high-order

node.lifting ()

node.vel_set ()$=$ vset_max

if mach $<$ mach_thr and node.

vel_set ()$==$ vset_max :

//node changes to low-order

node.projection()

node.vel_set ()$=$ vset_min 
[1] N. Frapolli, S. S. Chikatamarla, and I. V. Karlin, Entropic lattice Boltzmann model for compressible flows, Phys. Rev. E 92, 061301(R) (2015).

[2] B. Dorschner, S. S. Chikatamarla, and I. V. Karlin, Entropic multirelaxation-time lattice Boltzmann method for moving and deforming geometries in three dimensions, Phys. Rev. E 95, 063306 (2017).

[3] A. Mazloomi M, S. S. Chikatamarla, and I. V. Karlin, Entropic Lattice Boltzmann Method for Multiphase Flows, Phys. Rev. Lett. 114, 174502 (2015).

[4] M. Wöhrwag, C. Semprebon, A. Mazloomi Moqaddam, I. Karlin, and H. Kusumaatmaja, Ternary Free-Energy Entropic Lattice Boltzmann Model with a High Density Ratio, Phys. Rev. Lett. 120, 234501 (2018).

[5] X. Shan, X.-F. Yuan, and H. Chen, Kinetic theory representation of hydrodynamics: a way beyond the Navier-Stokes equation, J. Fluid Mech. 550, 413 (2006).

[6] X. Shan and X. He, Discretization of the Velocity Space in the Solution of the Boltzmann Equation, Phys. Rev. Lett. 80, 65 (1998).

[7] S. S. Chikatamarla and I. V. Karlin, Entropy and Galilean Invariance of Lattice Boltzmann Theories, Phys. Rev. Lett. 97, 190601 (2006).

[8] S. S. Chikatamarla and I. V. Karlin, Lattices for the lattice Boltzmann method, Phys. Rev. E 79, 046701 (2009).

[9] G. Di Ilio, B. Dorschner, G. Bella, S. Succi, and I. V. Karlin, Simulation of turbulent flows with the entropic multirelaxation time lattice Boltzmann method on body-fitted meshes, J. Fluid Mech. 849, 35 (2018).

[10] A. Krämer, K. Küllmer, D. Reith, W. Joppich, and H. Foysi, Semi-Lagrangian off-lattice Boltzmann method for weakly compressible flows, Phys. Rev. E 95, 023305 (2017).

[11] S. Ansumali, I. V. Karlin, S. Arcidiacono, A. Abbas, and N. I. Prasianakis, Hydrodynamics beyond Navier-Stokes: Exact Solution to the Lattice Boltzmann Hierarchy, Phys. Rev. Lett. 98, 124502 (2007).

[12] J. Meng and Y. Zhang, Accuracy analysis of high-order lattice Boltzmann models for rarefied gas flows, J. Comput. Phys. 230, 835 (2011).

[13] M. Mendoza, B. M. Boghosian, H. J. Herrmann, and S. Succi, Fast Lattice Boltzmann Solver for Relativistic Hydrodynamics, Phys. Rev. Lett. 105, 014502 (2010).

[14] Y. H. Qian and S. A. Orszag, Lattice BGK models for the Navier-Stokes equation: Nonlinear deviation in compressible regimes, Europhys. Lett. 21, 255 (1993).

[15] Y.-H. Qian and Y. Zhou, Complete Galilean-invariant lattice BGK models for the Navier-Stokes equation, Europhys. Lett. 42, 359 (1998).

[16] N. I. Prasianakis and I. V. Karlin, Lattice Boltzmann method for thermal flow simulation on standard lattices, Phys. Rev. E 76, 016702 (2007).

[17] M. H. Saadat, F. Bösch, and I. V. Karlin, Lattice Boltzmann model for compressible flows on standard lattices: Variable Prandtl number and adiabatic exponent, Phys. Rev. E 99, 013306 (2019).

[18] M. H. Saadat, S. A. Hosseini, B. Dorschner, and I. V. Karlin, Extended lattice Boltzmann model for gas dynamics, Phys. Fluids 33, 046104 (2021).
[19] M. H. Saadat, B. Dorschner, and I. Karlin, Extended lattice Boltzmann model, Entropy 23, 475 (2021).

[20] B. Dorschner, F. Bösch, and I. V. Karlin, Particles on Demand for Kinetic Theory, Phys. Rev. Lett. 121, 130602 (2018).

[21] E. Reyhanian, B. Dorschner, and I. V. Karlin, Thermokinetic lattice Boltzmann model of nonideal fluids, Phys. Rev. E 102, 020103(R) (2020).

[22] F. J. Alexander, S. Chen, and J. D. Sterling, Lattice Boltzmann thermohydrodynamics, Phys. Rev. E 47, R2249 (1993).

[23] T. Kataoka and M. Tsutahara, Lattice Boltzmann model for the compressible Navier-Stokes equations with flexible specificheat ratio, Phys. Rev. E 69, 035701(R) (2004).

[24] Q. Li, Y. L. He, Y. Wang, and W. Q. Tao, Coupled doubledistribution-function lattice Boltzmann method for the compressible Navier-Stokes equations, Phys. Rev. E 76, 056705 (2007).

[25] M. Watari, Finite difference lattice Boltzmann method with arbitrary specific heat ratio applicable to supersonic flow simulations, Physica A: Statistical Mechanics and its Applications 382, 502 (2007).

[26] S. H. Kim, H. Pitsch, and I. D. Boyd, Slip velocity and Knudsen layer in the lattice Boltzmann method for microscale flows, Phys. Rev. E 77, 026704 (2008).

[27] J. Meng and Y. Zhang, Gauss-Hermite quadratures and accuracy of lattice Boltzmann models for nonequilibrium gas flows, Phys. Rev. E 83, 036704 (2011).

[28] A. N. Gorban, N. Kazantzis, Y. G. Kevrekidis, H. C. Öttinger, and K. Theodoropoulos, Model Reduction and Coarse-Graining Approaches for Multiscale Phenomena (Springer-Verlag, Berlin Heidelberg, 2006).

[29] G. Di Staso, H. J. H. Clercx, S. Succi, and F. Toschi, DSMCLBM mapping scheme for rarefied and non-rarefied gas flows, J. Comput. Sci. 17, 357 (2016).

[30] G. Di Staso, H. J. H. Clercx, S. Succi, and F. Toschi, Lattice Boltzmann accelerated direct simulation Monte Carlo for dilute gas flow simulations, Philos. Trans. R. Soc. A 374, 20160226 (2016).

[31] J. E. Broadwell, Study of rarefied shear flow by the discrete velocity method, J. Fluid Mech. 19, 401 (1964).

[32] L. Mieussens, Discrete velocity model and implicit scheme for the BGK equation of rarefied gas dynamics, Math. Models Methods Appl. Sci. 10, 1121 (2000).

[33] V. A. Titarev, Efficient deterministic modelling of threedimensional rarefied gas flows, Commun. Comput. Phys. 12, 162 (2012).

[34] C. Baranger, J. Claudel, N. Hérouard, and L. Mieussens, Locally refined discrete velocity grids for stationary rarefied flow simulations, J. Comput. Phys. 257, 572 (2014).

[35] R. R. Arslanbekov, V. I. Kolobov, and A. A. Frolova, Kinetic solvers with adaptive mesh in phase space, Phys. Rev. E 88, 063301 (2013).

[36] V. V. Aristov, O. V. Ilyin, and O. A. Rogozin, Kinetic multiscale scheme based on the discrete-velocity and lattice-Boltzmann methods, J. Comput. Sci. 40, 101064 (2020).

[37] O. V. Ilyin, A method for simulating the dynamics of rarefied gas based on lattice Boltzmann equations and 
the BGK equation, Comput. Math. Math. Phys. 58, 1817 (2018).

[38] J. Meng, Y. Zhang, and X. Shan, Multiscale lattice Boltzmann approach to modeling gas flows, Phys. Rev. E 83, 046701 (2011).

[39] D. Wilde, A. Krämer, D. Reith, and H. Foysi, Semi-lagrangian lattice Boltzmann method for compressible flows, Phys. Rev. E 101, 053306 (2020).

[40] C. Shu, X. D. Niu, and Y. T. Chew, Taylor-series expansion and least-squares-based lattice Boltzmann method: Two-dimensional formulation and its applications, Phys. Rev. E 65, 036708 (2002).

[41] M. Cheng and K. C. Hung, Lattice Boltzmann method on nonuniform mesh, Int. J. Comput. Eng. Sci. 05, 291 (2004).

[42] A. Bardow, I. V. Karlin, and A. A. Gusev, General characteristic-based algorithm for off-lattice Boltzmann simulations, Europhys. Lett. 75, 434 (2006).
[43] B. Dorschner, N. Frapolli, S. S. Chikatamarla, and I. V. Karlin, Grid refinement for entropic lattice Boltzmann models, Phys. Rev. E 94, 053311 (2016).

[44] G. Wissocq, J.-F. Boussuge, and P. Sagaut, Consistent vortex initialization for the athermal lattice Boltzmann method, Phys. Rev. E 101, 043306 (2020).

[45] Anderson John D, Modern Compressible Flow: With Historical Perspective (McGraw-Hill, New York, United States, 1990).

[46] G. A. Bird, Molecular Gas Dynamics and the Direct Simulation of Gas Flows, The Oxford Engineering Science Series (Clarendon Press, Oxford, United Kingdom, 1994).

[47] T. Ohwada, Structure of normal shock waves: Direct numerical analysis of the Boltzmann equation for hard-sphere molecules, Phys. Fluids A: Fluid Dynamics 5, 217 (1993).

[48] C. Cercignani, Theory and Application of the Boltzmann Equation (Scottish Academic Press, Edinburgh, 1975).

[49] S. Ansumali and I. V. Karlin, Kinetic boundary conditions in the lattice Boltzmann method, Phys. Rev. E 66, 026311 (2002). 